\title{
The use of fish and herptiles in traditional folk therapies in three districts of Chenab riverine area in Punjab, Pakistan
}

\author{
Muhammad Altaf ${ }^{1^{*}}$ D, Arshad Mehmood Abbasi ${ }^{2^{*}}$, Muhammad Umair ${ }^{3}$, Muhammad Shoaib Amjad ${ }^{4}$, \\ Kinza Irshad ${ }^{2}$ and Abdul Majid Khan ${ }^{5}$
}

\begin{abstract}
Background: Like botanical taxa, various species of animals are also used in traditional and modern health care systems. Present study was intended with the aim to document the traditional uses of herptile and fish species among the local communities in the vicinity of the River Chenab, Punjab Pakistan.
\end{abstract}

Method: Data collected by semi-structured interviews and questionnaires were subsequently analyzed using relative frequency of citation $(F C)$, fidelity level $(F L)$, relative popularity level $(R P L)$, similarity index $(\mathrm{SI})$, and rank order priority $(\mathrm{ROP})$ indices.

Results: Out of total 81 reported species, ethnomedicinal uses of eight herptiles viz. Aspideretes gangeticus, A. hurum, Eublepharis macularius, Varanus bengalensis, Python molurus, Eryx johnii, Ptyas mucosus mucosus, Daboia russelii russelii and five fish species including Hypophthalmichthys molitrix, Cirrhinus reba, Labeo dero, Mastacembelus armatus, and Pethia ticto were reported for the first time from this region. Fat, flesh, brain, and skin were among the commonly utilized body parts to treat allergy, cardiovascular, nervous and respiratory disorders, sexual impotency, skin infections, and as antidote and anti-diabetic agents. Hoplobatrachus tigerinus, Duttaphrynus stomaticus, and Ptyas mucosus mucosus (herptiles), as well as Labeo rohita, Wallago attu, and Cirrhinus reba (fish) were top ranked with maximum informant reports, frequency of citations, and rank order priority. Uromastyx hardwickii,

Ctenopharyngodon idella, H. molitrix, Cirrhinus mrigala, C. reba, L. rohita, L. calbasu, L. dero, and Pethia ticto were the species with 100\% fidelity level. Furthermore, medicinal uses of Aspideretes gangeticus, Aspideretes hurum, Calotes versicolor, Daboia russelii russelii, Hypophthalmichthys molitrix, Cirrhinus reba, Labeo dero, Mastacembelus armatus, Pethia ticto, and Gagata cenia were reported for the first time.

Conclusion: About half of the reported species depicted zero similarity index with previously reported literature, which indicates strong associations of local inhabitants with animal species, particularly for therapeutic purpose. Inclusive studies on composition and bioactivities of the species with maximum use reports may contribute significantly in animal-based novel drugs discovery.

Keywords: Ethnozoology, Medicinal, Herptile, Fish, Punjab, Pakistan

\footnotetext{
*Correspondence: altaf_mughal450@yahoo.com; arshad799@yahoo.com

'Department of Zoology, Women University of Azad Jammu and Kashmir, Bagh, Pakistan

${ }^{2}$ Department of Environment Sciences, COMSATS University Islamabad, Abbottabad Campus, Abbottabad 22060, Pakistan

Full list of author information is available at the end of the article
}

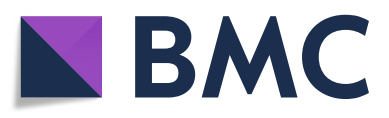

( The Author(s). 2020 Open Access This article is licensed under a Creative Commons Attribution 4.0 International License, which permits use, sharing, adaptation, distribution and reproduction in any medium or format, as long as you give appropriate credit to the original author(s) and the source, provide a link to the Creative Commons licence, and indicate if changes were made. The images or other third party material in this article are included in the article's Creative Commons licence, unless indicated otherwise in a credit line to the material. If material is not included in the article's Creative Commons licence and your intended use is not permitted by statutory regulation or exceeds the permitted use, you will need to obtain permission directly from the copyright holder. To view a copy of this licence, visit http://creativecommons.org/licenses/by/4.0/. The Creative Commons Public Domain Dedication waiver (http://creativecommons.org/publicdomain/zero/1.0/) applies to the data made available in this article, unless otherwise stated in a credit line to the data. 


\section{Introduction}

The multipurpose usage of animal species, e.g., as food, medicine, entertainment, magic, music and religion, tools for art, and in trade, is well known [1-9]. It has been reported that loving, watching, and working with animal species is beneficial to lower heartbeat and control stroke [10]. Animal-based products are used as traditional medicines, and an estimated $8.7 \%$ of the vital chemicals used in modern healthcare systems are extracted from different animal species [11]. However, compared to plant species, animal-based products are widely neglected [12]. Inhabitants of rural areas are more depended on animal-based products as food and medicines, and possess significant traditional knowledge of zootherapies $[9,11,13]$. Many species of animals, either wild or domesticated, are important to humans [13]. Wild animal species are often under threat due to anthropogenic activities like illegal hunting and trade for food, medicines, and ornamental purposes, deforestation, agriculture intensification, urbanization, and industrialization [3, 14-19].

Herptiles and fish are recognized as extremely fascinating and important animal species [20-22]. In many societies, different species of herptiles and fish are used in ethnomedicine and folklore to treat health disorders [16, 22,23 . An estimated 10,450 species of reptiles and 7850 amphibian species have been reported [24] globally. In Pakistan, 195 species of reptiles [25] and 24 species of amphibians [26] have been documented so far. The Asian region has also a high diversity of marine and freshwater fish species (22907 and 10036 species, respectively) [24, 27]. An estimated 186 species of freshwater fish and 719 species of marine water fish have been reported so far in Pakistan [28]. However, traditional uses of animal species, particularly to treat diseases in humans and other animals, have rarely been documented in Pakistan [6, 7, 29-32]. To best of our knowledge, traditional uses of herptiles and fish species have never been reported before in Pakistan. Therefore, the present study was planned to document herptiles and fish species used to treat various diseases by the local communities residing along the Chenab riverine areas, i.e., Gujranwala, Gujrat, and Sialkot districts in the Punjab province of Pakistan. Qualitative indices were used to elucidate commonly utilized species with high fidelity level and frequency of citation. We hope the data provided will be of significant value for pharmaceutical industries to discover animal-based novel drugs to meet the recent challenges to human health.

\section{Materials and methods}

\section{Study area}

The River Chenab is the combination of two main streams, i.e., Chandra and Bhaga originating from the Himalayan region of Himachal Pradesh in India. After passing through the Siwalik Range in the south, and the Lesser Himalayas in the north of Indian Jammu and Kashmir, it continues into Pakistan [33]. The present study was conducted in three districts of the Chenab riverine area, i.e., Gujranwala, Gujrat, and Sialkot (Fig. 1) from March 2016 to April 2017. The study area covers $9830 \mathrm{~km}^{2}$, with temperature ranges from around $0{ }^{\circ} \mathrm{C}$ in December to $50{ }^{\circ} \mathrm{C}$ in June [34-36]. This region has a high diversity of wild fauna, comprising 150 species of birds, 47 herptiles, 34 fish, and 15 mammalian species $[15,37-39]$. Demographically almost $52 \%$ of the population are male, and $48 \%$ are female. The major population is rural, and encompasses Arain, Gujjar, Jutt, Sheikh, Rana Butt, Malik, and Mughal casts. Punjabi is the common language spoken, although some people speak Siraiki and Urdu, while educated people can also speak English to some extent [34-36].

\section{Data collection and analysis}

Following the Nagoya Protocol, prior informed consent was taken from local informants for data collection and publication. In addition, the International Society of Ethnobiology Code of Ethics (http://www.ethnobiology.net/) was also followed. Ethnomedicinal uses of herptiles and fish species along with cultural importance were collected from local informants $(n=100)$ using semistructured interviews and group discussions. Informants including farmers, fishermen, hunters, teachers, and health practitioners were selected based on their traditional knowledge of animal species, i.e., herptiles and fish. Animals were identified using "The Amphibian and Reptiles of Pakistan" [25], and "Freshwater Fish of Pakistan" were also consulted for correct classification and identification of fish of the study area [40]. Different indices, i.e., relative frequency of citation (RFC), fidelity level (FL), relative popularity level (RPL), rank order priority (ROP) and similarity index (SI), were used to analyze that data.

Relative frequency of mention (RFC) was calculated using formula as reported previously [41].

$$
\mathrm{RFC}=\frac{\mathrm{FC}}{\mathrm{N}}(0 \leq \mathrm{RFC} \leq 1)
$$

Where FC is the frequency of citation for an ethnomedicinal or cultural use of a specific species and $N$ is the total number of informants.

Fidelity level (FL) was obtained using the method explained earlier [42] based on formula

$$
\mathrm{FL}(\%)=\mathrm{N}_{\mathrm{p}} / \mathrm{FC} \times 100
$$

where $N_{\mathrm{p}}$ indicates number of informants reporting major ailment for a specific species of herptiles or fish 


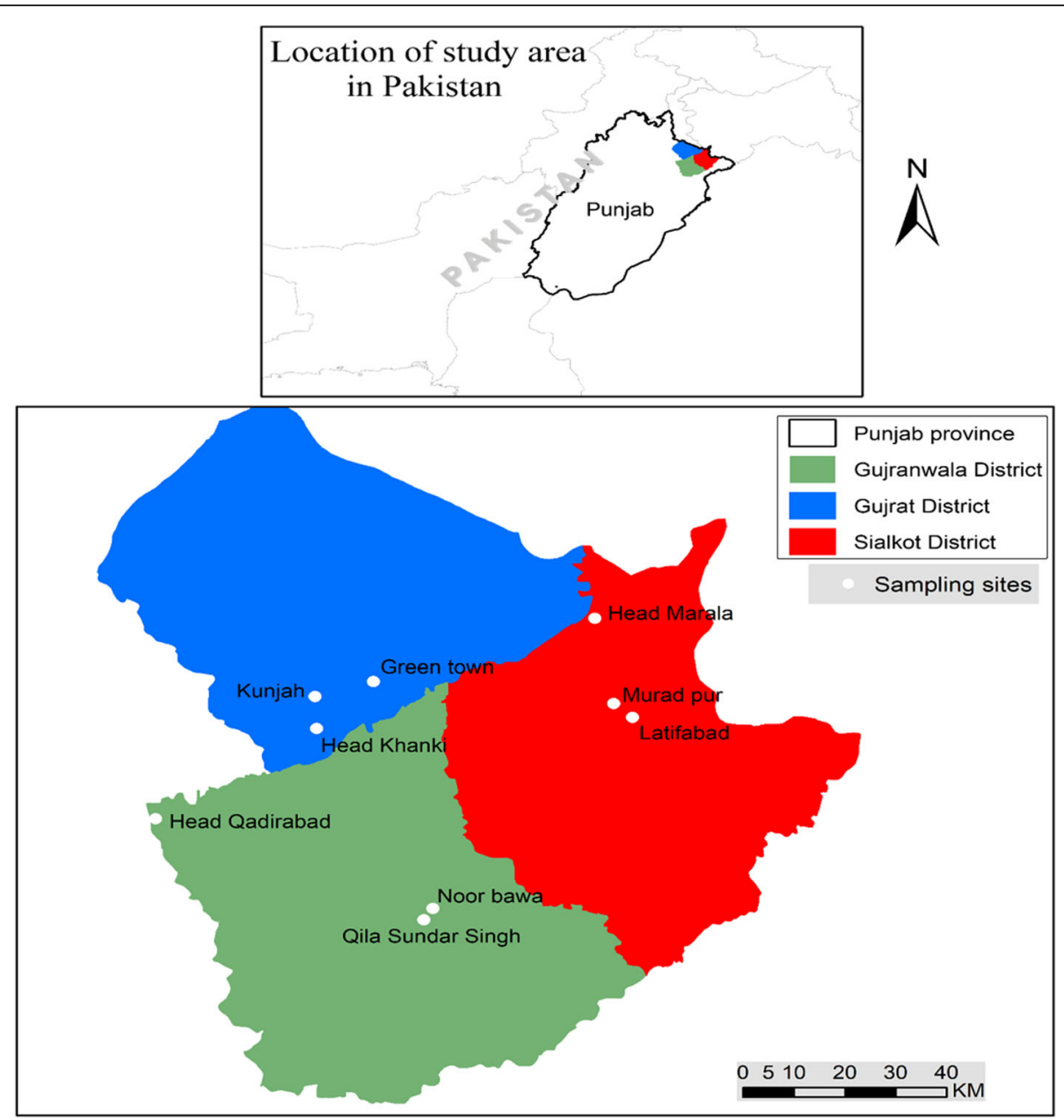

Fig. 1 Map of study area with survey sites

and FC is the frequency of citation for ethno-medicinal or cultural use of that species.

Relative popularity level (RPL) of the reported species was elucidated as reported by [43, 44]. Herptiles and fish species were classified into two groups (i) "popular" and (ii) "unpopular." Popular herptiles and fish species were those having more than half of the maximum frequency of citation (FC), whereas the left-over herptiles and fish were documented as unpopular. For popular herptiles and fish species, a horizontal line was imaginary, namely the average number of uses per species is independent of the frequency of citation (FC), who recognizes the herptiles and fish; therefore, the average numeral of uses of a popular herptile and fish species does not enhance with the add to frequency of citations who cite a herptile and fish for any medical use. For the popular herptiles and fish, the RPL was chosen to one (1). For herptiles and fish in the unpopular group, the relative popularity level value is less than 1.0 .
Rank order priority (ROP) is used to grade plants and animal species and was calculated as explained earlier $[43,44]$ and was analyzed by the following formula

$$
\mathrm{ROPs}=\mathrm{FL} \times \mathrm{RPL}
$$

Similarity index (SI) was calculated as reported previously [3]

$$
\mathrm{SI}=M_{\mathrm{s}} / M_{t} \quad(0 \leq \mathrm{SI} \leq 1)
$$

$M_{\mathrm{s}}=$ Alike number of medicinal uses in the previous and present research records for a specific herptiles and fish species. $M_{\mathrm{t}}=$ Total number of medicinal uses in the present research reports for a specific herptiles and fish species.

Principal component analysis (PCA). Data were statistically analyzed with the help of principal component analysis by using Past software Version 3 [45]. 


\section{Results and discussion Demography}

Data were collected from 100 informants of an age between 18 and 75 years (Fig. 2). About 70\% informants were literate, and participants had finished having primary, matric, intermediate, bachelors, and master levels $(23,24,21,8$, and 3 , respectively). The majority of the informants (76\%) were from rural areas with agricultural background.

\section{Local nomenclature}

Vernacular names of animal species are usually based on environment, myths, morphological characteristics, habitat, and social associations of species with humans. As mentioned in Table 1, "daddo" is used as suffix in six animals (13\%) of the reported herpetiles such as Bufotes latastii (chitkbra daddo), Duttaphrynus stomaticus (ghariallo daddo), Microhyla ornate (bona daddo), Fejervarya limnocharis (pidda daddo), Hoplobatrachus tigerinus (wada daddo), and Sphaerotheca breviceps (chota dahri daddo). Variations in the vernacular names of these animals are due morphological differences, e.g., H. tigerinus has a larger size and was called "wada daddo." Similarly, M. ornata has a smaller size and was called "bona daddo." A very small frog was called "pidda daddo," while B. latastii, which has patches on body, was called "chitkbra daddo," and S. breviceps, Amphiesma stolatum, and Ophisops jerdonii, which all have lines on the body, were named as "chota dahri daddo."
Likewise, 14 species of lizards had the suffix "kirli" such as Laudakia melanura melanura (kali kirli), Eublepharis macularius (korh kirli), Cyrtopodion montiumsalsorum (sahrai kirli), Cyrtopodion Scabrum (toor Kirli), Hemidactylus flaviviridis (gharailo kirli), Hemidactylus persicus (Irani kirli), Acanthodactylus cantoris (neeli poosh kirli), Ophisops jerdonii (safaid dahari kirli), Ablepharus grayanus (bahri kirli), Ablepharus pannonicus (surakh posh kirli), Eutropis macularia (bori kaa kirli), Eurylepis taeniolatus taeniolatus (maidani kirli), Ophiomorus tridactylus (tray ungl kirli), and Scincella himalayana (pahari kirli). Fifteen species of snakes had the suffix "sap," e.g., Leptotyphlops macrorhynchus (dhaga sap), Ramphotyphlops braminus (dhaga sap), Python molurus (azdha sap), Amphiesma stolatum (lakeer dhari sap), Boiga trigonata (billi sap), Lytorhynchus paradoxus (ollu sap), Oligodon arnensis arnensis (kukri sap), Oligodon taeniolatus taeniolatus (kukri sap), Platyceps rhodorachis rhodorachis (Pheesi sap), Psammophis leithii leithii (teer maar sap), Psammophis schokari schokari (saharai sap), Ptyas mucosus mucosus (chohay-maar sap), Xenochrophis piscator piscator (chitra sap), Daboia russelii russelii (dabian wala sap), and Echis carinatus sochureki (pathar sap). Only two local names included "kukri sap" in the vernacular name (Oligodon arnensis arnensis and O. taeniolatus taeniolatus), and "dhaga sap" was the local name of Leptotyphlops macrorhynchus and Ramphotyphlops braminus. Local nomenclature of snakes was also based on their external morphology

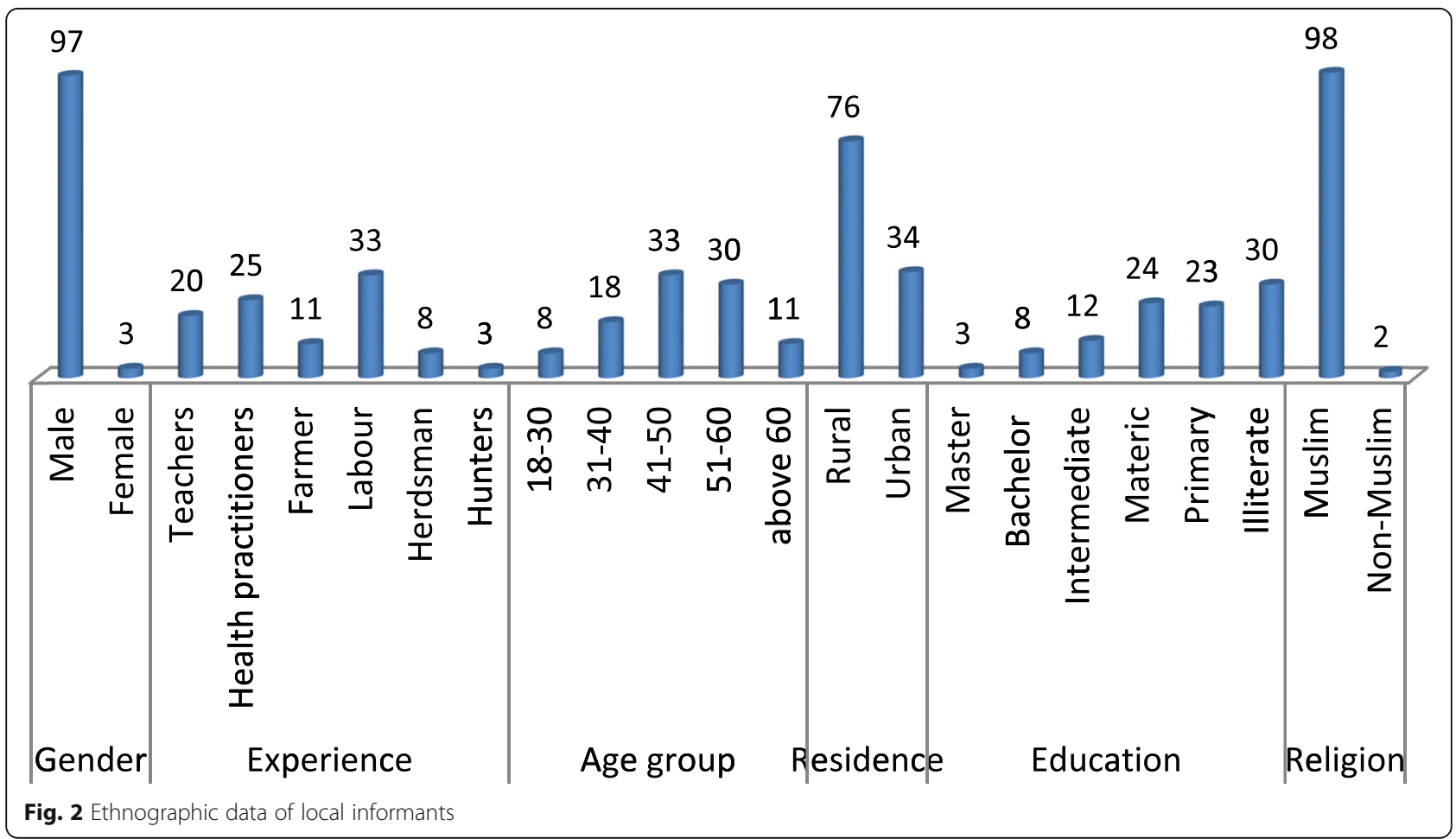




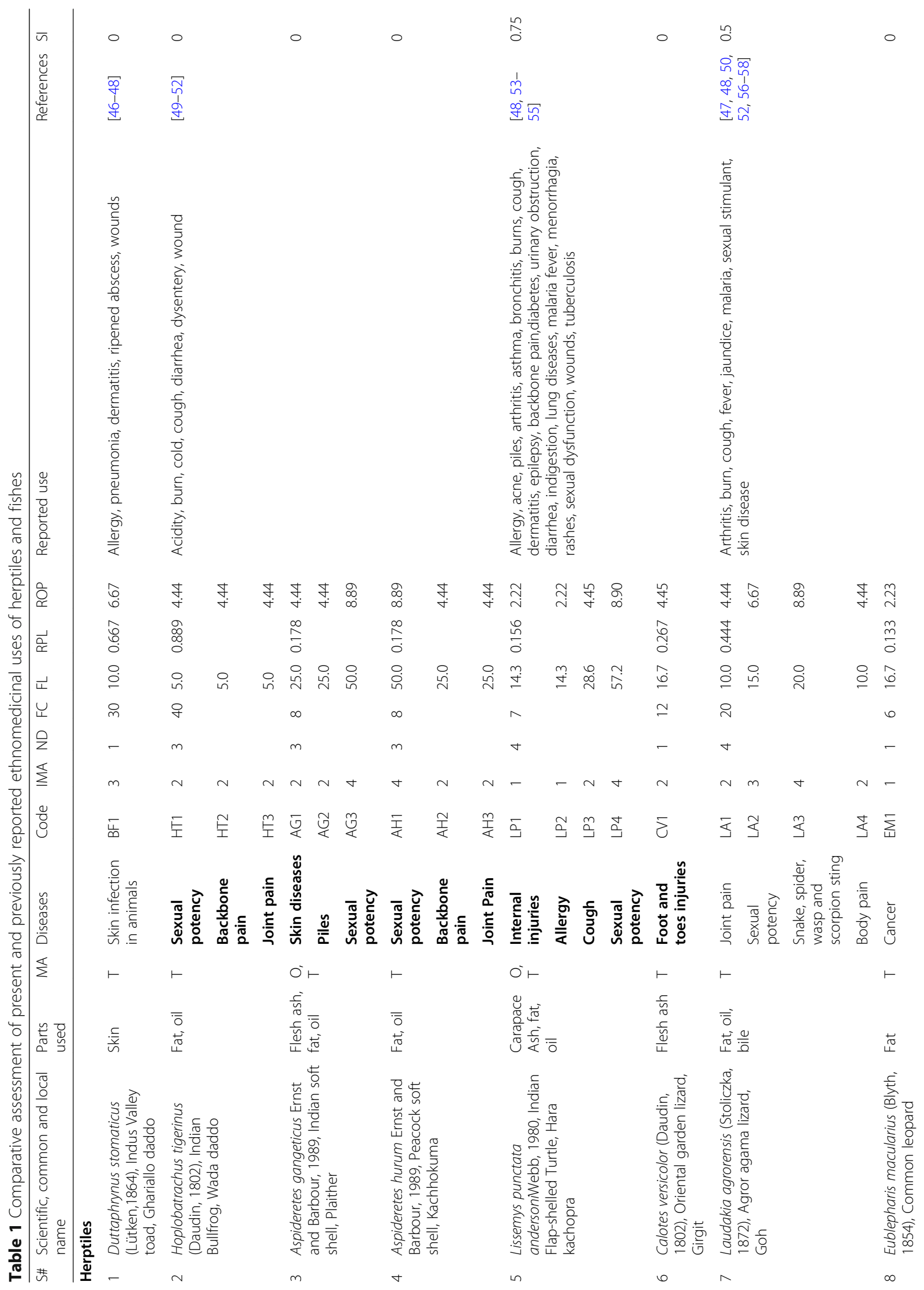




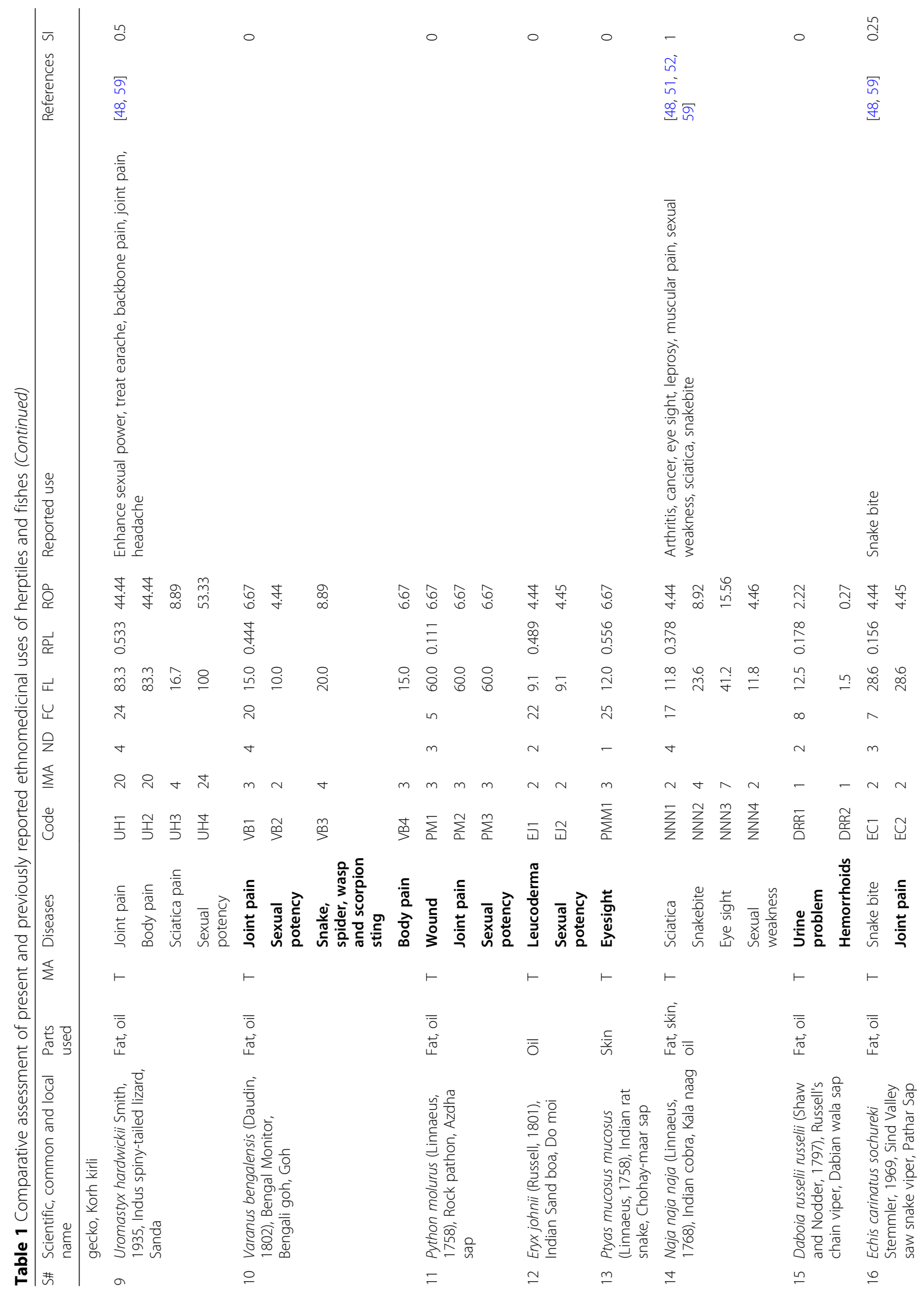




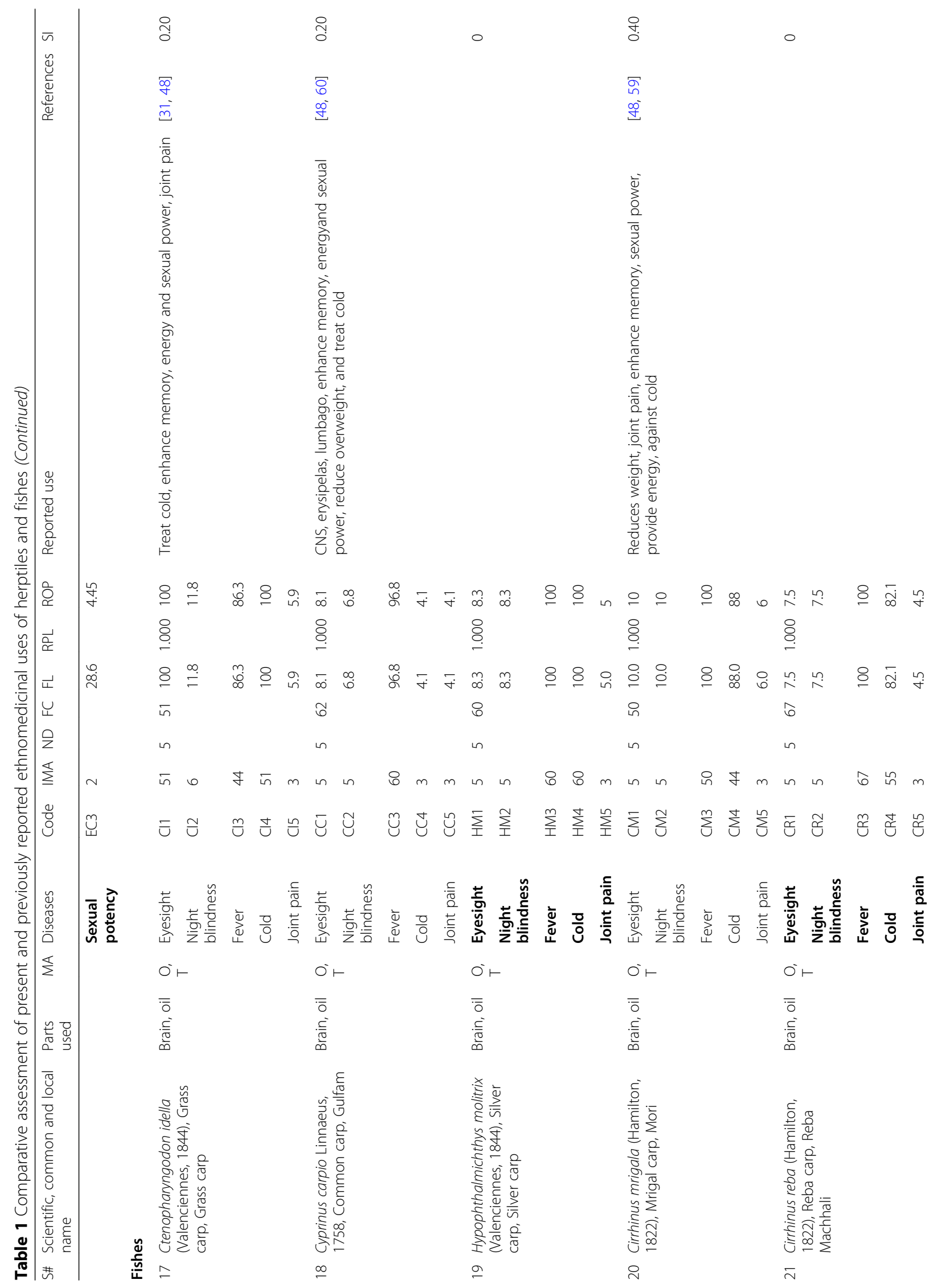




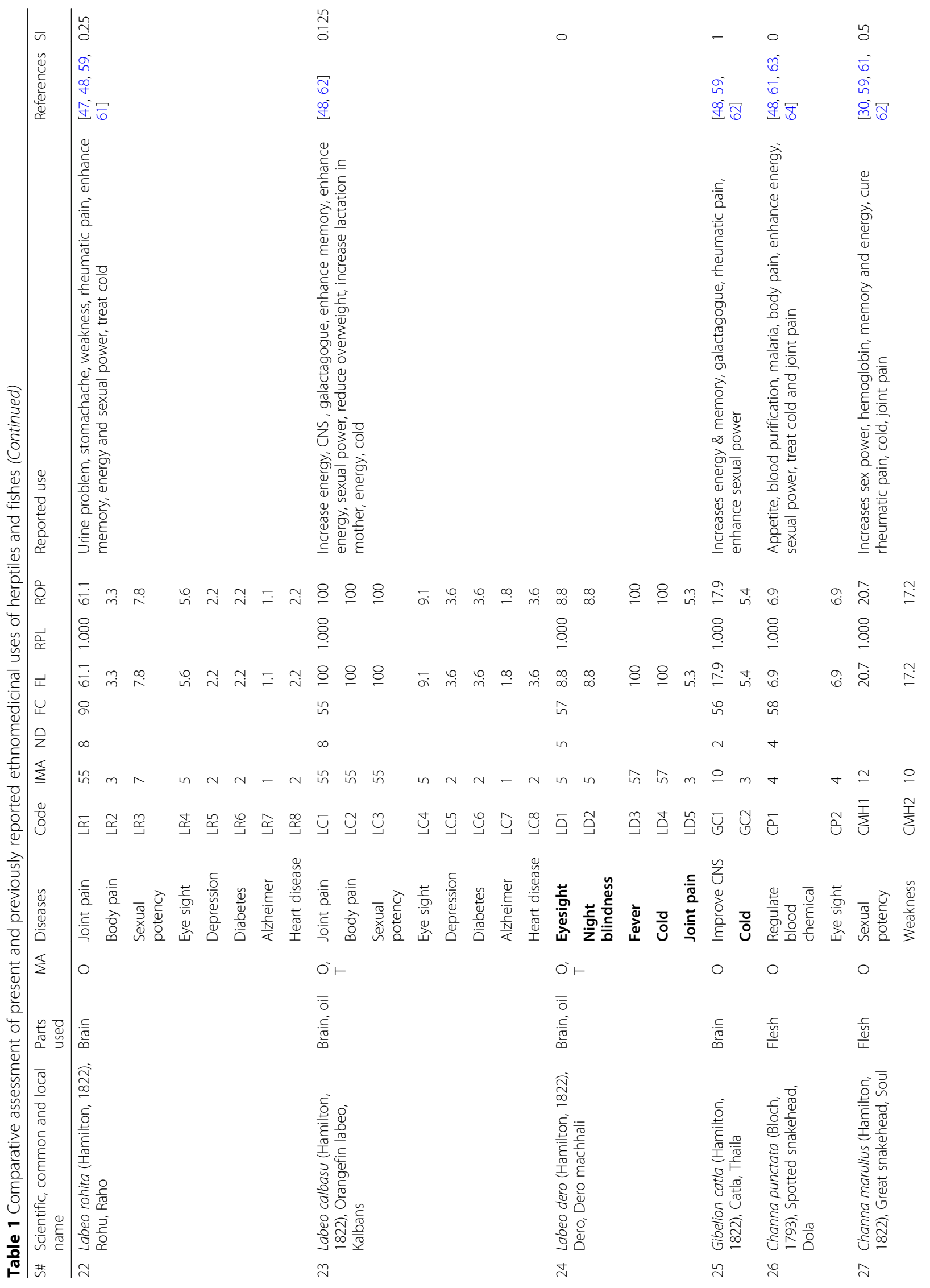




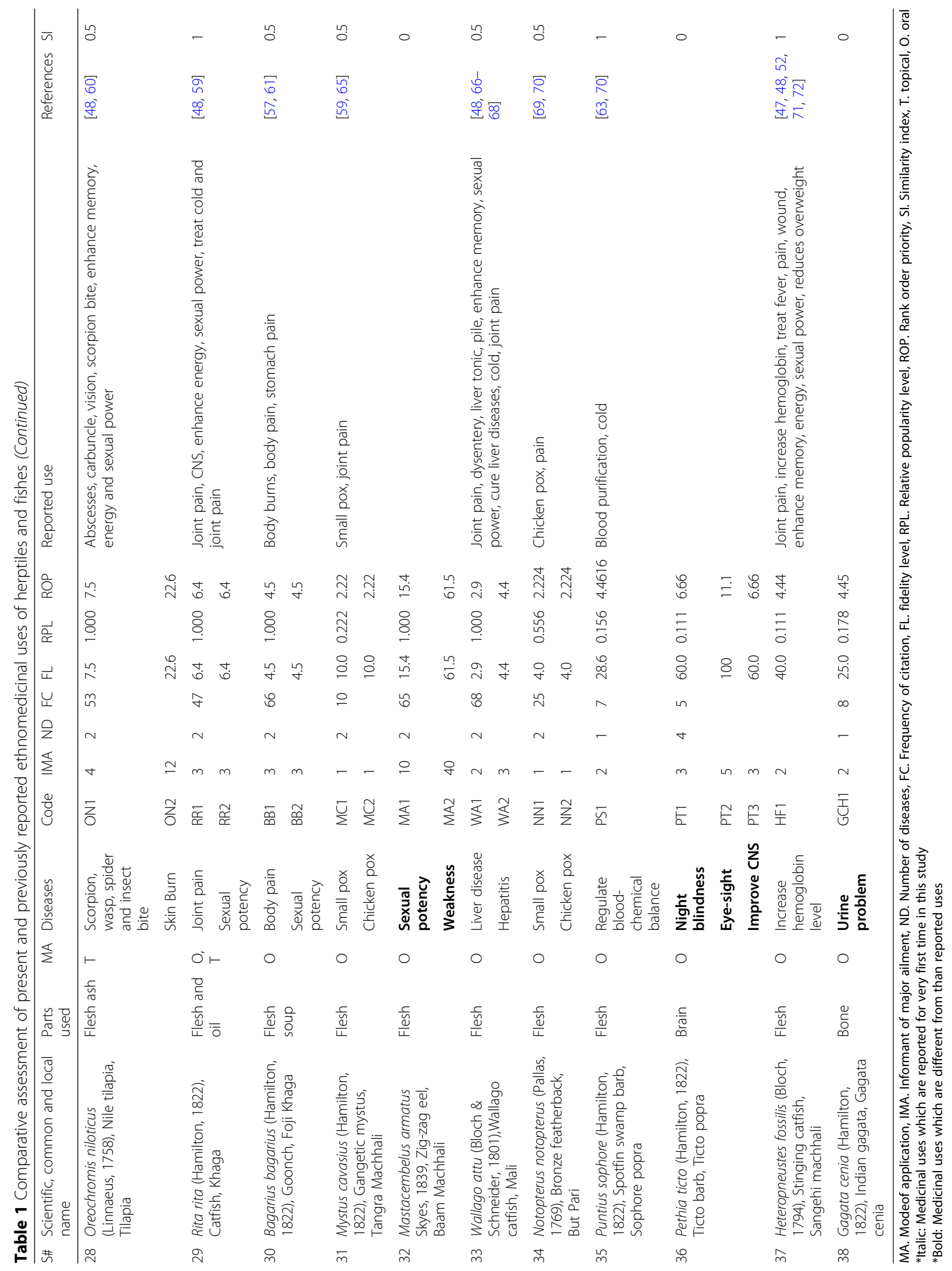


such as L. melanura melanura, A. cantoris, A. pannonicus, Naja naja naja, and E. macularia which have black, blue, red, and black and brown color lines and hence were named kali kirli, neeli poosh kirli, surakh posh kirli, Kala naag, and bori kaa kirli, respectively.

The vernacular names of the reported species had also connections with the habitats like sahrai kirli as the name of $C$. montiumsalsorum, because it lives in desert (Sahari) landscapes, whereas H. flaviviridis and B. stomaticus were named asghariallo daddo and ghariallo kirli, respectively as they live in houses (ghar) or their vicinity. S. himalayanais was called pahari kirli because it inhabits mountain areas (pahar). Likewise, vernacular names had also a connection with the morphology of species, O. tridactylus, e.g., has three tows and is known as "tray ungl kirli," L. macrorhynchus and R. braminus snakes are very thin and are known as dhaga sap.

Eight species of fish had the same suffix "machhali" such as Cirrhinus reba (reba machhali), Labeo dero (dero machhali), Oreochromis niloticus (tilapia/chira machhli), Mystus cavasius (tangra machhali), Mastacembelus armatus (baam machhali), Osteobrama cotio (pali roo machhali), Salmostoma bacaila (choti chal machhali), and Heteropneustes fossilis (sangehi machhali). The English and local names of Hypophthalmichthys molitrix were the same-"silver carp". Vernacular names of two species were based on their color: H. molitrix has silver color and $C$. idella has grass color; therefore, they were named as silver carp and grass carp, respectively. Some fish were also classified on the basis of morphology, e.g., the shape of Channa punctata is similar to an arm muscle; therefore, it was called dola (bicep muscle); $O$. niloticus size resembles a house sparrow andthus, the species was named chira machhli (chira is house sparrow) and S. bacaila has small size, and was known as choti chal machhali (choti means small).

\section{Myths about herptiles and fish}

Some common myths on snake and fish species were also noted during the field survey. These myths were comparable to previous reports [15].

- It is a common assumption that if Eryx johnii (common sand boa) bites somebody, it will bite on the arrival of the new moon in each month.

- If the male or female ofa snake pair is killed by someone, then the other will certainly take revenge from the assassin.

- Naja naja changes into a human after 100 year of age and can transfer poison to a person if it sniffs someone.

- Some people of the study area believe that Python molurus (rock python) has seven mouths.
- Most people believe that a special bone called Mankana is present in the snake head, and that this bone can absorb poison from snake-bitten people.

- Seeing a snake in a dream means that an enemy may attack a person.

- Every species of fish has a special kind head like a human and body like a fish, which locally known as Jal Pari.

- Turtles have a blade which can cut anything.

\section{Ethnomedicinal uses herptiles and fish}

Inhabitants of the study area possessed significant knowledge on the medicinal as well as cultural uses of animal species, particularly that of herptiles and fish (Fig. 3). As mentioned in Table 1, 38 species of herptiles and fish were used to treat various health disorders such as allergy, cardiovascular, nervous and respiratory disorders, sexual impotency, skin infections, and as antidote and anti-diabetic agents in human and livestock (Table 2).

\section{Body part(s) used}

In both herptiles and fish, as shown in Fig. 4, fat was the most commonly utilized body part and was used in the preparation of 19 recipes to treat a number of diseases, followed by flesh, brain, and skin, used in 14, 11, and 3 recipes, respectively. In the present study, the application of body fat of herptiles and fish served to treat rheumatic problems, eye diseases, sexual impotency, cold, fever, skin infections, nervous and cardiovascular diseases, diabetes, respiratory tract infections, urine problem, liver infections, heal wounds, and as antidote. This might be related to the presence of health beneficial metabolites, i.e., omega-3 fatty and omega- 6 fatty acids, etc. It has been reported that these acids contribute substantially in the treatment of neurological disorders, thrombotic, atherosclerosis, and act as anti-aging agents [73]. Beneficial effects of omega-3 fatty acids on atherosclerosis are mainly through their actions on plasma lipids. Likewise, their role in the reduction of blood pressure and plasminogen activator inhibitor, improvement of metabolic syndrome, and maintain endothelial function may be due to other potential antiatherogenic factors. As atherosclerosis is inflammatory diseases, omega-3 fatty acids offer protection through their anti-inflammatory effects [74-76]. The major benefit of omega- 3 fatty acids in patients with type 2 diabetes mellitus is the enhancement in their highly atherogenic lipid profile. It has been reported less development of pancreatic islet-cell autoimmunity and reduction in insulin resistance was observed in mice treated with omega-3 fatty acids $[75,77]$.

Water-soluble vitamins [78], $\alpha 1$ and $\alpha 2$ collagen (I) proteins [79], and different amino acids, i.e., 4-hydroxyproline, aspartic acid, threonine, serine, glutamic acid, proline, glycine, alanine, cysteine, valine, methionine, methionine, 


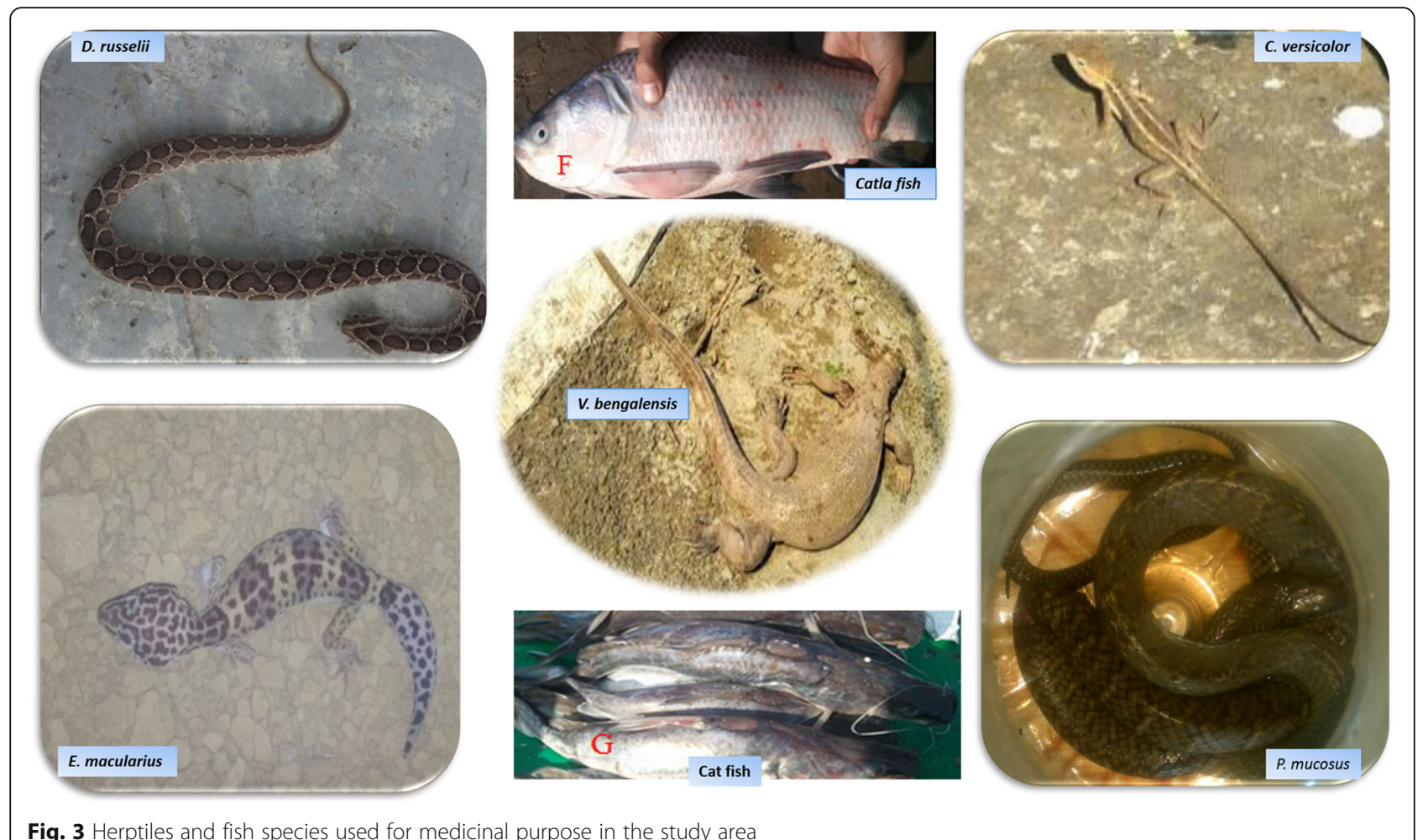

Fig. 3 Herptiles and fish species used for medicinal purpose in the study area

leucine, tyrosine, phenylalanine, histidine, lysine, arginine, and hydroxylysine [80], have also been reported in the skin of fish. Collagen (I) is used in membranes for guided tissue regeneration [81]. While, essential amino acids profile of fish is required to humans for balanced diet [82].

Inhabitants of the study area use carapace of the turtle to treat internal injuries, allergy, cough, and as a sexual stimulant. These pharmacological properties of turtle carapace are mainly due to the presence of $\beta$-pleated sheet keratin [83], keratinocytes, melanocytes, lipids [84], and mineralized collagen fibrils [85]. Likewise, fish brain is used to treat joint pain, Alzheimer, heart diseases, for sexual potency, to improve eye-sight, as an anti-depressant and anti-diabetic. The health beneficial properties of fish brain mainly attributed to rich compounds of docosahexaenoic acid, omega-3 fatty acids, and proteins present in it $[76,86]$.

\section{Diseases treated}

As mentioned in Fig. 5, joint pain, eye diseases, sexual impotency, common cold, and fever were among the top ranked diseases treated with maximum number of animal-based recipes. Lack of hygiene, nutritional deficiency, and "community evils" were among the major factors involved in the high prevalence of diseases in the study area. Comparative analysis of the present findings with previous reports on medicinal uses of herptiles and fish species indicate that different methods of treatments and body parts were used in study area (Table 1). The inhabitants of the study area use skin of Indus valley toad (Duttaphrynus stomaticus) to treat skin infections, while the same species have been reported to treat allergy, thelitis, bolianerengia, bronchial pneumonia, dermatitis, abscess, and to heal wounds [46-48]. Body fat of the Indian bullfrog (Hoplobatrachus tigerinus) was used to treat backbone pain, sexual impotency, and joint pain; but in previous studies [49-52], different body parts of this species have been reported against acidity, burn, cold, cough, diarrhea, dysentery, and to heal wounds. In the study area, carapace ash, fat, and oil of the Indian flap-shelled turtle (Lissemys punctata andersoni) were used to enhance sexual potency and in the treatment of internal injuries, allergy, and cough. However, this species has also been reported to treat acne, piles, arthritis, asthma, bronchitis, burns, cough, dermatitis, epilepsy, backbone pain, diabetes, urinary obstruction, diarrhea, indigestion, lung diseases, malaria fever, menorrhagia, rashes, wound healing, and tuberculosis [48, 53-55]. Fat, oil, and bile of Agror agama (Laudakia agrorensis) were used for joint pain; sexual potency; snake, spider, wasp, and scorpion sting; as well as body pain. Same species are used to treat jaundice, joint pain, malaria, arthritis, burn, cough, fever, and skin disease [47, 48, 50, 52, 56-58]. The Indus spiny-tail lizard (Uromastyx hardwickii) is used in the treatment of body pain, joint pain, sciatica pain, and for sexual potency, 
Table 2 Cultural uses of herptiles and fishes in the study area

\begin{tabular}{|c|c|c|c|c|c|c|c|c|c|c|c|c|c|c|c|c|}
\hline Sr. \# & Scientific, common, and local name & FC & RFC & $\mathrm{CU}$ & STS & $\mathrm{MD}$ & NR & CC & $\mathrm{TL}$ & ET & FD & $\mathrm{HF}$ & MG & EX & OR & SPR \\
\hline 1 & $\begin{array}{l}\text { Bufotes latastii (Boulenger, 1882), Ladakh } \\
\text { Toad, Chitkbra daddo }\end{array}$ & 7 & 0.401 & 2 & LC & $x$ & $\sqrt{ }$ & $x$ & $x$ & $x$ & $x$ & $\sqrt{ }$ & $x$ & $x$ & $x$ & $x$ \\
\hline 2 & $\begin{array}{l}\text { Duttaphrynus stomaticus (Lütken, 1864)," } \\
\text { Indus Valley toad, Ghariallo daddo }\end{array}$ & 30 & 1.720 & 3 & LC & $\sqrt{ }$ & $\sqrt{ }$ & $x$ & $x$ & $x$ & $x$ & $\sqrt{ }$ & $x$ & $x$ & $x$ & $x$ \\
\hline 3 & $\begin{array}{l}\text { Microhyla ornata (Dumerila and Bibron, 1841), } \\
\text { Ant Frog, Bona daddo }\end{array}$ & 7 & 0.401 & 2 & LC & $x$ & $\sqrt{ }$ & $x$ & $x$ & $x$ & $x$ & $\sqrt{ }$ & $x$ & $x$ & $x$ & $x$ \\
\hline 4 & $\begin{array}{l}\text { Fejervarya limnocharis (Gravenhorst, 1829), Asian } \\
\text { Grass Frog, Pidda daddo }\end{array}$ & 5 & 0.287 & 2 & LC & $x$ & $\sqrt{ }$ & $x$ & $x$ & $x$ & $x$ & $\sqrt{ }$ & $x$ & $x$ & $x$ & $x$ \\
\hline 5 & $\begin{array}{l}\text { Hoplobatrachus tigerinus (Daudin, 1802), Indian } \\
\text { Bullfrog, Wada daddo }\end{array}$ & 40 & 2.294 & 4 & LC & $\sqrt{ }$ & $\sqrt{ }$ & $\sqrt{ }$ & $x$ & $x$ & $x$ & $\sqrt{ }$ & $x$ & $x$ & $x$ & $x$ \\
\hline 6 & $\begin{array}{l}\text { Sphaerotheca breviceps (Schneider, 1799), Indian } \\
\text { burrowing frog, Chota dahri daddo }\end{array}$ & 5 & 0.287 & 4 & LC & $x$ & $\sqrt{ }$ & $x$ & $x$ & $x$ & $x$ & $\sqrt{ }$ & $x$ & $x$ & $x$ & $x$ \\
\hline 7 & $\begin{array}{l}\text { Aspideretes gangeticus Ernst and Barbour, 1989, } \\
\text { Indian soft shell, Plaither }\end{array}$ & 8 & 0.459 & 5 & $\mathrm{VU}$ & $\sqrt{ }$ & $\sqrt{ }$ & $\sqrt{ }$ & $x$ & $x$ & $x$ & $\sqrt{ }$ & $x$ & $x$ & $x$ & $\sqrt{ }$ \\
\hline 8 & $\begin{array}{l}\text { Aspideretes hurum Ernst and Barbour, 1989, } \\
\text { Peacock soft shell, Kachhokuma }\end{array}$ & 8 & 0.459 & 5 & $\mathrm{VU}$ & $\sqrt{ }$ & $\sqrt{ }$ & $\sqrt{ }$ & $x$ & $x$ & $x$ & $\sqrt{ }$ & $x$ & $x$ & $x$ & $\sqrt{ }$ \\
\hline 9 & $\begin{array}{l}\text { Lissemys punctata andersoni Webb, 1980, Indian } \\
\text { Flap-shelled Turtle, Hara kachopra }\end{array}$ & 7 & 0.401 & 5 & LC & $\sqrt{ }$ & $\sqrt{ }$ & $\sqrt{ }$ & $x$ & $x$ & $x$ & $\sqrt{ }$ & $x$ & $x$ & $x$ & $\sqrt{ }$ \\
\hline 10 & $\begin{array}{l}\text { Calotes minor (Hardwicke and gray, 1827), } \\
\text { Hardwicke's Short Tail Agama, Choti dum kirli }\end{array}$ & 5 & 0.287 & 2 & $\mathrm{NE}$ & $x$ & $x$ & $x$ & $x$ & $x$ & $x$ & $\sqrt{ }$ & $x$ & $x$ & $x$ & $\sqrt{ }$ \\
\hline 11 & $\begin{array}{l}\text { Calotes versicolor (Daudin, 1802), Oriental } \\
\text { garden lizard, Girgit }\end{array}$ & 12 & 0.688 & 3 & $\mathrm{NE}$ & $\sqrt{ }$ & $x$ & $x$ & $x$ & $x$ & $x$ & $\sqrt{ }$ & $x$ & $x$ & $x$ & $\sqrt{ }$ \\
\hline 12 & $\begin{array}{l}\text { Laudakia agrorensis (Stoliczka, 1872), } \\
\text { Agror agama, Goh }\end{array}$ & 20 & 1.147 & 4 & $\mathrm{NE}$ & $\sqrt{ }$ & $x$ & $x$ & $\sqrt{ }$ & $x$ & $x$ & $\sqrt{ }$ & $x$ & $x$ & $x$ & $\sqrt{ }$ \\
\hline 13 & $\begin{array}{l}\text { Laudakia melanura melanura (Blyth, 1854), } \\
\text { Black agama, Kali kirli }\end{array}$ & 2 & 0.115 & 2 & $\mathrm{NE}$ & $x$ & $x$ & $x$ & $x$ & $x$ & $x$ & $\sqrt{ }$ & $x$ & $x$ & $x$ & $\sqrt{ }$ \\
\hline 14 & $\begin{array}{l}\text { Trapelus agilis pakistanensis Rastegar-Pouyani, 1999, } \\
\text { Brilliant ground agama, Korh kirla }\end{array}$ & 5 & 0.287 & 2 & $\mathrm{NE}$ & $x$ & $x$ & $x$ & $x$ & $x$ & $x$ & $\sqrt{ }$ & $x$ & $x$ & $x$ & $\sqrt{ }$ \\
\hline 15 & $\begin{array}{l}\text { Eublepharis macularius (Blyth, 1854), Common } \\
\text { leopard gecko, Korh kirli }\end{array}$ & 6 & 0.344 & 4 & $\mathrm{NE}$ & $\sqrt{ }$ & $x$ & $x$ & $x$ & $x$ & $x$ & $\sqrt{ }$ & $x$ & $x$ & $x$ & $\sqrt{ }$ \\
\hline 16 & $\begin{array}{l}\text { Cyrtopodion montiumsalsorum (Annandale, 1913), } \\
\text { Salt range ground gecko, Sahrai kirli }\end{array}$ & 4 & 0.229 & 2 & $\mathrm{NE}$ & $x$ & $x$ & $x$ & $x$ & $x$ & $x$ & $\sqrt{ }$ & $x$ & $x$ & $x$ & $\sqrt{ }$ \\
\hline 17 & $\begin{array}{l}\text { Cyrtopodion Scabrum (Heydenn 1827), Common } \\
\text { tuberculated ground gecko, Toor kirli }\end{array}$ & 2 & 0.115 & 2 & LC & $x$ & $x$ & $x$ & $x$ & $x$ & $x$ & $\sqrt{ }$ & $x$ & $x$ & $x$ & $\sqrt{ }$ \\
\hline 18 & $\begin{array}{l}\text { Hemidactylus flaviviridis Rüppell, 1835, Yellow } \\
\text { belly common house gecko, Gharailo kirli }\end{array}$ & 44 & 2.523 & 2 & $\mathrm{NE}$ & $x$ & $x$ & $x$ & $x$ & $x$ & $x$ & $\sqrt{ }$ & $x$ & $x$ & $x$ & $\sqrt{ }$ \\
\hline 19 & $\begin{array}{l}\text { Hemidactylus persicus Anderson, 1872, Persian } \\
\text { house gecko, Irani kirli }\end{array}$ & 3 & 0.172 & 2 & $\mathrm{NE}$ & $x$ & $x$ & $x$ & $x$ & $x$ & $x$ & $\sqrt{ }$ & $x$ & $x$ & $x$ & $\sqrt{ }$ \\
\hline 20 & $\begin{array}{l}\text { Acanthodactylus cantoris Gunther, 1864, Blue } \\
\text { tailed sand lizard, Neeli poosh kirli }\end{array}$ & & 0.000 & 2 & $\mathrm{NE}$ & $x$ & $x$ & $x$ & $x$ & $x$ & $x$ & $\sqrt{ }$ & $x$ & $x$ & $x$ & $\sqrt{ }$ \\
\hline 21 & $\begin{array}{l}\text { Ophisops jerdonii Blyth, 1853, Punjab snake-eyed } \\
\text { lacerta, Safaid dahari kirli }\end{array}$ & 4 & 0.229 & 2 & $\mathrm{NE}$ & $x$ & $x$ & $x$ & $x$ & $x$ & $x$ & $\sqrt{ }$ & $x$ & $x$ & $x$ & $\sqrt{ }$ \\
\hline 22 & $\begin{array}{l}\text { Ablepharus grayanus (Stoliczka, 1872), Earless } \\
\text { snake eyed skink, Bahri kirli }\end{array}$ & 1 & 0.057 & 2 & $\mathrm{NE}$ & $x$ & $x$ & $x$ & $x$ & $x$ & $x$ & $\sqrt{ }$ & $x$ & $x$ & $x$ & $\sqrt{ }$ \\
\hline 23 & $\begin{array}{l}\text { Ablepharus pannonicus (Fitzinger, 1824), Red } \\
\text { tail snake eyed skink, Surakh posh kirli }\end{array}$ & 3 & 0.172 & 2 & $\mathrm{NE}$ & $x$ & $x$ & $x$ & $x$ & $x$ & $x$ & $\sqrt{ }$ & $x$ & $x$ & $x$ & $\sqrt{ }$ \\
\hline 24 & $\begin{array}{l}\text { Eutropis macularia (Blyth, 1853), Bronz grass skink, } \\
\text { Bori kaa kirli }\end{array}$ & 2 & 0.115 & 2 & $\mathrm{NE}$ & $x$ & $x$ & $x$ & $x$ & $x$ & $x$ & $\sqrt{ }$ & $x$ & $x$ & $x$ & $\sqrt{ }$ \\
\hline 25 & $\begin{array}{l}\text { Eurylepis taeniolatus taeniolatus Blyth, 1854, Alpine } \\
\text { Punjab skink, Maidani kirli }\end{array}$ & 3 & 0.172 & 2 & $\mathrm{NE}$ & $x$ & $x$ & $x$ & $x$ & $x$ & $x$ & $\sqrt{ }$ & $x$ & $x$ & $x$ & $\sqrt{ }$ \\
\hline 26 & $\begin{array}{l}\text { Ophiomorus tridactylus(Blyth, 1853), Three toed } \\
\text { snake skink, Tray ungl kirli }\end{array}$ & 2 & 0.115 & 2 & $\mathrm{NE}$ & X & $x$ & $x$ & $x$ & $x$ & $x$ & $\sqrt{ }$ & $x$ & $x$ & $x$ & $\sqrt{ }$ \\
\hline 27 & Scincella himalayana (Günther, 1864), Himalayan & 2 & 0.115 & 2 & $\mathrm{NE}$ & $x$ & $x$ & $x$ & $x$ & $x$ & $x$ & $\sqrt{ }$ & $x$ & $x$ & $x$ & $\sqrt{ }$ \\
\hline
\end{tabular}


Table 2 Cultural uses of herptiles and fishes in the study area (Continued)

\begin{tabular}{|c|c|c|c|c|c|c|c|c|c|c|c|c|c|c|c|c|}
\hline Sr. \# & Scientific, common, and local name & FC & RFC & $\mathrm{CU}$ & STS & $\mathrm{MD}$ & NR & $\mathrm{CC}$ & $\mathrm{TL}$ & ET & FD & $\mathrm{HF}$ & MG & EX & OR & $\mathrm{SH}$ \\
\hline 28 & $\begin{array}{l}\text { Uromastyx hardwickii Smith,1935, Indus spiny-tail } \\
\text { lizard, Sanda }\end{array}$ & 24 & 1.376 & 3 & $\mathrm{NE}$ & $\sqrt{ }$ & $x$ & $x$ & $x$ & $x$ & $x$ & $\sqrt{ }$ & $x$ & $x$ & $x$ & $\sqrt{ }$ \\
\hline 29 & $\begin{array}{l}\text { Varanus bengalensis (Daudin, 1802), Bengal Monitor, } \\
\text { Bengali goh, Goh }\end{array}$ & 20 & 1.147 & 4 & LC & $\sqrt{ }$ & $x$ & $x$ & $\sqrt{ }$ & $x$ & $x$ & $\sqrt{ }$ & $x$ & $x$ & $x$ & $\sqrt{ }$ \\
\hline 30 & $\begin{array}{l}\text { Leptotyphlops macrorhynchus Hahn, 1978, long-nosed } \\
\text { worm snake, Dhaga sap }\end{array}$ & 5 & 0.287 & 2 & LC & $x$ & $\sqrt{ }$ & $x$ & $x$ & $x$ & $x$ & $\sqrt{ }$ & $x$ & $x$ & $x$ & $x$ \\
\hline 31 & $\begin{array}{l}\text { Ramphotyphlops braminus Daudin, 1803, Barhminy } \\
\text { blind snake, Dhaga sap }\end{array}$ & 3 & 0.172 & 2 & NE & $x$ & $\sqrt{ }$ & $x$ & $x$ & $x$ & $x$ & $\sqrt{ }$ & $x$ & $x$ & $x$ & $x$ \\
\hline 32 & Python molurus (Linnaeus, 1758), Rock pathon, Azdha sap & 5 & 0.287 & 4 & $\mathrm{VU}$ & $\sqrt{ }$ & $\sqrt{ }$ & $x$ & $x$ & $\sqrt{ }$ & $x$ & $\sqrt{ }$ & $x$ & $x$ & $x$ & $x$ \\
\hline 33 & $\begin{array}{l}\text { Amphiesma stolatum (Linnaeus, 1758), Buff Striped } \\
\text { Keelback, Lakeer dhari sap }\end{array}$ & 5 & 0.287 & 3 & NE & $x$ & $\sqrt{ }$ & $x$ & $x$ & $\sqrt{ }$ & $x$ & $\sqrt{ }$ & $x$ & $x$ & $x$ & $x$ \\
\hline 34 & $\begin{array}{l}\text { Boiga trigonata (Schneider, 1802), Common cat snake, } \\
\text { Billi sap }\end{array}$ & 5 & 0.287 & 2 & LC & $x$ & $\sqrt{ }$ & $x$ & $x$ & $x$ & $x$ & $\sqrt{ }$ & $x$ & $x$ & $x$ & $x$ \\
\hline 35 & $\begin{array}{l}\text { Lytorhynchus paradoxus (Gunther, 1875), Sind longnose } \\
\text { sand snake, Ollu sap }\end{array}$ & 6 & 0.344 & 2 & $\mathrm{NE}$ & $x$ & $\sqrt{ }$ & $x$ & $x$ & $x$ & $x$ & $\sqrt{ }$ & $x$ & $x$ & $x$ & $x$ \\
\hline 36 & $\begin{array}{l}\text { Oligodon arnensis arnensis (Shaw, 1802), Banded kukri } \\
\text { snake, Kukri Sap }\end{array}$ & 6 & 0.344 & 2 & $N E$ & $x$ & $\sqrt{ }$ & $x$ & $x$ & $x$ & $x$ & $\sqrt{ }$ & $x$ & $x$ & $x$ & $x$ \\
\hline 37 & $\begin{array}{l}\text { Oligodon taeniolatus taeniolatus (Jerdon, 1853), Streaked } \\
\text { kukri snake, Kukri sap }\end{array}$ & 4 & 0.229 & 2 & LC & $x$ & $\sqrt{ }$ & $x$ & $x$ & $x$ & $x$ & $\sqrt{ }$ & $x$ & $x$ & $x$ & $x$ \\
\hline 38 & $\begin{array}{l}\text { Platyceps rhodorachis rhodorachis (Jan, 1865), Cliff racer, } \\
\text { Pheesi sap }\end{array}$ & 5 & 0.287 & 2 & $\mathrm{NE}$ & $x$ & $\sqrt{ }$ & $x$ & $x$ & $x$ & $x$ & $\sqrt{ }$ & $x$ & $x$ & $x$ & $x$ \\
\hline 39 & Eryx johnii(Russell, 1801), Common Sand boa, Do moi & 22 & 1.261 & 3 & NE & $\sqrt{ }$ & $\sqrt{ }$ & $x$ & $x$ & $x$ & $x$ & $\sqrt{ }$ & $x$ & $x$ & $x$ & $x$ \\
\hline 40 & $\begin{array}{l}\text { Psammophis leithii leithii Günther, 1869, Steppe ribbon } \\
\text { snake, Teer maar sap }\end{array}$ & 8 & 0.459 & 2 & NE & $x$ & $\sqrt{ }$ & $x$ & $x$ & $x$ & $x$ & $\sqrt{ }$ & $x$ & $x$ & $x$ & $x$ \\
\hline 41 & $\begin{array}{l}\text { Psammophis schokari schokari (Forskal, 1775), Schokari } \\
\text { sand racer snake, Saharai sap }\end{array}$ & 5 & 0.287 & 2 & NE & $x$ & $\sqrt{ }$ & $x$ & $x$ & $x$ & $x$ & $\sqrt{ }$ & $x$ & $x$ & $x$ & $x$ \\
\hline 42 & $\begin{array}{l}\text { Ptyas mucosus mucosus (Linnaeus, 1758), Indian rat snake, } \\
\text { Chohay-maar sap }\end{array}$ & 25 & 1.433 & 4 & $N E$ & $\sqrt{ }$ & $\sqrt{ }$ & $x$ & $x$ & $\sqrt{ }$ & $x$ & $\sqrt{ }$ & $x$ & $x$ & $x$ & $x$ \\
\hline 43 & $\begin{array}{l}\text { his piscator piscator (Schneider, 1799), Chekered } \\
\text { Chitra sap }\end{array}$ & 8 & 0.459 & 2 & $\mathrm{NE}$ & $x$ & $\sqrt{ }$ & $x$ & $x$ & $x$ & $x$ & $\sqrt{ }$ & $x$ & $x$ & $x$ & $x$ \\
\hline 44 & hneider, 1801), Common & 12 & 0.688 & 2 & $\mathrm{NE}$ & $x$ & $\sqrt{ }$ & $x$ & $x$ & $x$ & $x$ & $\sqrt{ }$ & $x$ & $x$ & $x$ & $x$ \\
\hline 45 & Naja naja naja (Linnaeus, 1768), Indian cobra, Kala naag & 17 & 0.975 & 5 & LC & $\sqrt{ }$ & $\sqrt{ }$ & $x$ & $x$ & $\sqrt{ }$ & $x$ & $\sqrt{ }$ & $\sqrt{ }$ & $x$ & $x$ & $x$ \\
\hline 46 & $\begin{array}{l}\text { Daboia russelii russelii (Shaw and Nodder, 1797), Russell's } \\
\text { chain viper, Dabian wala sap }\end{array}$ & 8 & 0.459 & 3 & NE & $\sqrt{ }$ & $\sqrt{ }$ & $x$ & $x$ & $x$ & $x$ & $\sqrt{ }$ & $x$ & $x$ & $x$ & \\
\hline 47 & $\begin{array}{l}\text { Echis carinatus sochureki Stemmler, 1964, Sind } \\
\text { snake viper, Pathar Sap }\end{array}$ & 7 & 0.401 & 3 & NE & $\sqrt{ }$ & $\sqrt{ }$ & $x$ & $x$ & $x$ & $x$ & $\sqrt{ }$ & $x$ & $x$ & $x$ & $x$ \\
\hline 48 & $\begin{array}{l}\text { aryngodon idella } \\
\text { rp }\end{array}$ & 51 & 2.924 & 6 & NE & $\sqrt{ }$ & $\sqrt{ }$ & $\sqrt{ }$ & $\sqrt{ }$ & $\sqrt{ }$ & $\sqrt{ }$ & $x$ & $x$ & $x$ & $x$ & $x$ \\
\hline 49 & bio (Linnaeus, 1758), Common carp, Gulfam & 62 & 3.555 & 6 & $\mathrm{VU}$ & $\sqrt{ }$ & $\sqrt{ }$ & $\sqrt{ }$ & $\sqrt{ }$ & $\sqrt{ }$ & $\sqrt{ }$ & $x$ & $x$ & $x$ & $x$ & $x$ \\
\hline 50 & $\begin{array}{l}\text { Hypophthalmichthys molitrix (Valenciennes, 1844), Silver } \\
\text { carp, Silver carp }\end{array}$ & 60 & 3.440 & 6 & NT & $\sqrt{ }$ & $\sqrt{ }$ & $\sqrt{ }$ & $\sqrt{ }$ & $\sqrt{ }$ & $\sqrt{ }$ & $x$ & $x$ & $x$ & $x$ & $x$ \\
\hline 51 & Cirrhinus mrigala (Hamilton, 1822), Mrigal carp, Mori & 50 & 2.867 & 6 & LC & $\sqrt{ }$ & $\sqrt{ }$ & $\sqrt{ }$ & $\sqrt{ }$ & $\sqrt{ }$ & $\sqrt{ }$ & $x$ & $x$ & $x$ & $x$ & $x$ \\
\hline 2 & Cirrhinus reba (Hamilton, 1822), Reba carp, Reba Machhali & 67 & 3.842 & 6 & LC & $\sqrt{ }$ & $\sqrt{ }$ & $\sqrt{ }$ & $\sqrt{ }$ & $\sqrt{ }$ & $\sqrt{ }$ & $x$ & $x$ & $x$ & $x$ & $x$ \\
\hline 53 & Labeo rohita (Hamilton, 1822), Rohu, Raho & 90 & 5.161 & 6 & LC & $\sqrt{ }$ & $\sqrt{ }$ & $\sqrt{ }$ & $\sqrt{ }$ & $\sqrt{ }$ & $\sqrt{ }$ & $x$ & $x$ & $x$ & $x$ & X \\
\hline 44 & Labeo calbasu (Hamilton, 1822), Orangefin labeo, Kalbans & 55 & 3.154 & 6 & LC & $\sqrt{ }$ & $\sqrt{ }$ & $\sqrt{ }$ & $\sqrt{ }$ & $\sqrt{ }$ & $\sqrt{ }$ & $x$ & $x$ & $x$ & $x$ & $x$ \\
\hline 55 & Labeo dero (Hamilton, 1822), Dero, Dero machhali & 57 & 3.268 & 6 & LC & $\sqrt{ }$ & $\sqrt{ }$ & $\sqrt{ }$ & $\sqrt{ }$ & $\sqrt{ }$ & $\sqrt{ }$ & $x$ & $x$ & $x$ & $x$ & $x$ \\
\hline 56 & Gibelion catla (Hamilton, 1822), Catla, Thaila & 56 & 3.211 & 6 & LC & $\sqrt{ }$ & $\sqrt{ }$ & $\sqrt{ }$ & $\sqrt{ }$ & $\sqrt{ }$ & $\sqrt{ }$ & $x$ & $x$ & $x$ & $x$ & $x$ \\
\hline D' & Channa punctata(Bloch, 1793), Spotted snakehead, Dola & 58 & 3.326 & 6 & LC & $\sqrt{ }$ & $\sqrt{ }$ & $\sqrt{ }$ & $\sqrt{ }$ & $\sqrt{ }$ & $\sqrt{ }$ & $x$ & $x$ & $x$ & $x$ & $x$ \\
\hline 58 & Channa marulius (Hamilton, 1822), Great snakehead, Soul & 58 & 3.326 & 6 & LC & $\sqrt{ }$ & $\sqrt{ }$ & $\sqrt{ }$ & $\sqrt{ }$ & $\sqrt{ }$ & $\sqrt{ }$ & $x$ & $x$ & $x$ & $x$ & $x$ \\
\hline 59 & Oreochromis niloticus (Linnaeus, 1758), Nile tilapia, Tilapia, & 53 & 3.039 & 6 & NE & $\sqrt{ }$ & $\sqrt{ }$ & $\sqrt{ }$ & $\sqrt{ }$ & $\sqrt{ }$ & $\sqrt{ }$ & $x$ & $x$ & $x$ & $x$ & \\
\hline
\end{tabular}


Table 2 Cultural uses of herptiles and fishes in the study area (Continued)

\begin{tabular}{|c|c|c|c|c|c|c|c|c|c|c|c|c|c|c|c|c|}
\hline Sr. \# & Scientific, common, and local name & FC & RFC & $\mathrm{CU}$ & STS & MD & NR & CC & $\mathrm{TL}$ & ET & $\mathrm{FD}$ & $\mathrm{HF}$ & MG & EX & OR & SPR \\
\hline 60 & Rita rita (Hamilton, 1822), Rita, Khaga & 47 & 2.695 & 6 & LC & $\sqrt{ }$ & $\sqrt{ }$ & $\sqrt{ }$ & $\sqrt{ }$ & $\sqrt{ }$ & $\sqrt{ }$ & $x$ & $x$ & $x$ & $x$ & $x$ \\
\hline 61 & Bagarius bagarius (Hamilton, 1822), Goonch, Foji Khaga & 66 & 3.784 & 6 & NT & $\sqrt{ }$ & $\sqrt{ }$ & $\sqrt{ }$ & $\sqrt{ }$ & $\sqrt{ }$ & $\sqrt{ }$ & $x$ & $x$ & $x$ & $x$ & $x$ \\
\hline 62 & $\begin{array}{l}\text { Mystus cavasius (Hamilton, 1822), Gangetic mystus, } \\
\text { Tangra machhali }\end{array}$ & 10 & 0.573 & 6 & LC & $\sqrt{ }$ & $\sqrt{ }$ & $\sqrt{ }$ & $\sqrt{ }$ & $\sqrt{ }$ & $\sqrt{ }$ & $x$ & $x$ & $x$ & $x$ & $x$ \\
\hline 63 & $\begin{array}{l}\text { Mastacembelus armatus (Lacepède, 1800), Zig-zag eel, } \\
\text { Baam machhali }\end{array}$ & 65 & 3.727 & 6 & LC & $\sqrt{ }$ & $\sqrt{ }$ & $\sqrt{ }$ & $\sqrt{ }$ & $\sqrt{ }$ & $\sqrt{ }$ & $x$ & $x$ & $x$ & $x$ & $x$ \\
\hline 64 & Sperata seenghala (Sykes, 1839), Giant river-catfish, Sangari & 62 & 3.555 & 5 & $\mathrm{NE}$ & $x$ & $\sqrt{ }$ & $\sqrt{ }$ & $\sqrt{ }$ & $\sqrt{ }$ & $\sqrt{ }$ & $x$ & $x$ & $x$ & $x$ & $x$ \\
\hline 65 & Wallago attu (Bloch \& Schneider, 1801), wallago catfish, Mali & 68 & 3.899 & 6 & NT & $\sqrt{ }$ & $\sqrt{ }$ & $\sqrt{ }$ & $\sqrt{ }$ & $\sqrt{ }$ & $\sqrt{ }$ & $x$ & $x$ & $x$ & $x$ & $x$ \\
\hline 66 & Eutropiichthys vacha (Hamilton, 1822), Batchwa vacha, Jhali & 56 & 3.211 & 5 & LC & $x$ & $\sqrt{ }$ & $\sqrt{ }$ & $\sqrt{ }$ & $\sqrt{ }$ & $\sqrt{ }$ & $x$ & $x$ & $x$ & $x$ & $x$ \\
\hline 67 & Tor macrolepis Heckel, 1838, Masheer, Masheer & 40 & 2.294 & 5 & $\mathrm{NE}$ & $x$ & $\sqrt{ }$ & $\sqrt{ }$ & $\sqrt{ }$ & $\sqrt{ }$ & $\sqrt{ }$ & $x$ & $x$ & $x$ & $x$ & $x$ \\
\hline 68 & Clupisoma garua (Hamilton, 1822), Garua bachcha, Bachhwa & 9 & 0.516 & 5 & LC & $x$ & $\sqrt{ }$ & $\sqrt{ }$ & $\sqrt{ }$ & $\sqrt{ }$ & $\sqrt{ }$ & $x$ & $x$ & $x$ & $x$ & $x$ \\
\hline 69 & Notopterus notopterus (Pallas, 1769), Bronze featherback, But Pari & 25 & 1.433 & 6 & LC & $\sqrt{ }$ & $\sqrt{ }$ & $\sqrt{ }$ & $\sqrt{ }$ & $\sqrt{ }$ & $\sqrt{ }$ & $x$ & $x$ & $x$ & $\sqrt{ }$ & $x$ \\
\hline 70 & Barilius modestusDay, 1872, Indus baril, Lahori Chalwa & 8 & 0.459 & 5 & NE & $x$ & $\sqrt{ }$ & $\sqrt{ }$ & $\sqrt{ }$ & $\sqrt{ }$ & $\sqrt{ }$ & $x$ & $x$ & $x$ & $x$ & $x$ \\
\hline 71 & $\begin{array}{l}\text { Puntius sophore (Hamilton, 1822), Spotfin swamp barb, } \\
\text { Sophore popra }\end{array}$ & 7 & 0.401 & 6 & LC & $\sqrt{ }$ & $\sqrt{ }$ & $\sqrt{ }$ & $\sqrt{ }$ & $\sqrt{ }$ & $\sqrt{ }$ & $x$ & $x$ & $x$ & $x$ & $x$ \\
\hline 72 & Pethia ticto (Hamilton, 1822), Ticto barb, Ticto popra & 5 & 0.287 & 6 & LC & $\sqrt{ }$ & $\sqrt{ }$ & $\sqrt{ }$ & $\sqrt{ }$ & $\sqrt{ }$ & $\sqrt{ }$ & $x$ & $x$ & $x$ & $x$ & $x$ \\
\hline 73 & $\begin{array}{l}\text { Parambassis ranga (Hamilton, 1822), Indian glassy fish, } \\
\text { Ranga sheesha }\end{array}$ & 8 & 0.459 & 5 & LC & $x$ & $\sqrt{ }$ & $\sqrt{ }$ & $\sqrt{ }$ & $\sqrt{ }$ & $\sqrt{ }$ & $x$ & $x$ & $x$ & $\sqrt{ }$ & $x$ \\
\hline 74 & Sisor rabdophorus Hamilton, 1822, Sisor catfish, Kirla machhali & 7 & 0.401 & 5 & LC & $x$ & $\sqrt{ }$ & $\sqrt{ }$ & $\sqrt{ }$ & $\sqrt{ }$ & $\sqrt{ }$ & $x$ & $x$ & $x$ & $x$ & $x$ \\
\hline 75 & $\begin{array}{l}\text { Xenentodon cancila (Hamilton, 1822), Freshwater needlefish, } \\
\text { Kaan Machhali }\end{array}$ & 5 & 0.287 & 5 & LC & $x$ & $\sqrt{ }$ & $\sqrt{ }$ & $\sqrt{ }$ & $\sqrt{ }$ & $\sqrt{ }$ & $x$ & $x$ & $x$ & $x$ & $x$ \\
\hline 76 & Garra gotyla (Gray, 1830), Gotyla, Pather Chat & 7 & 0.401 & 5 & LC & $x$ & $\sqrt{ }$ & $\sqrt{ }$ & $\sqrt{ }$ & $\sqrt{ }$ & $\sqrt{ }$ & $x$ & $x$ & $x$ & $x$ & $x$ \\
\hline 77 & Osteobrama cotio (Hamilton, 1822), Cotio, Pali roo machhali & 5 & 0.287 & 5 & LC & $x$ & $\sqrt{ }$ & $\sqrt{ }$ & $\sqrt{ }$ & $\sqrt{ }$ & $\sqrt{ }$ & $x$ & $x$ & $x$ & $x$ & $x$ \\
\hline 78 & $\begin{array}{l}\text { Salmostoma bacaila (Hamilton, 1822), Large razorbelly minnow, } \\
\text { Choti chal machhali }\end{array}$ & 6 & 0.344 & 5 & LC & $x$ & $\sqrt{ }$ & $\sqrt{ }$ & $\sqrt{ }$ & $\sqrt{ }$ & $\sqrt{ }$ & $x$ & $x$ & $x$ & $x$ & $x$ \\
\hline 79 & $\begin{array}{l}\text { Heteropneustes fossilis (Bloch, 1794), Stinging catfish, Sangehi } \\
\text { machhali }\end{array}$ & 5 & 0.287 & 6 & LC & $\sqrt{ }$ & $\sqrt{ }$ & $\sqrt{ }$ & $\sqrt{ }$ & $\sqrt{ }$ & $\sqrt{ }$ & $x$ & $x$ & $x$ & $x$ & $x$ \\
\hline 80 & Gagata cenia (Hamilton, 1822), Indian gagata, Gagata cenia & 8 & 0.459 & 6 & LC & $\sqrt{ }$ & $\sqrt{ }$ & $\sqrt{ }$ & $\sqrt{ }$ & $\sqrt{ }$ & $\sqrt{ }$ & $x$ & $x$ & $x$ & $x$ & $x$ \\
\hline 81 & Macrognathus pancalus Hamilton, 1822, Barred spiny eel, Garoj & 7 & 0.401 & 5 & LC & $x$ & $\sqrt{ }$ & $\sqrt{ }$ & $\sqrt{ }$ & $\sqrt{ }$ & $\sqrt{ }$ & $x$ & $x$ & $x$ & $x$ & $x$ \\
\hline
\end{tabular}

$M D$ medicinal, NR narrative, CC commercial, $T L$ tools, ET entertainment, FD food, HF harmful, OR ornamental, SPR superstitious, STS status

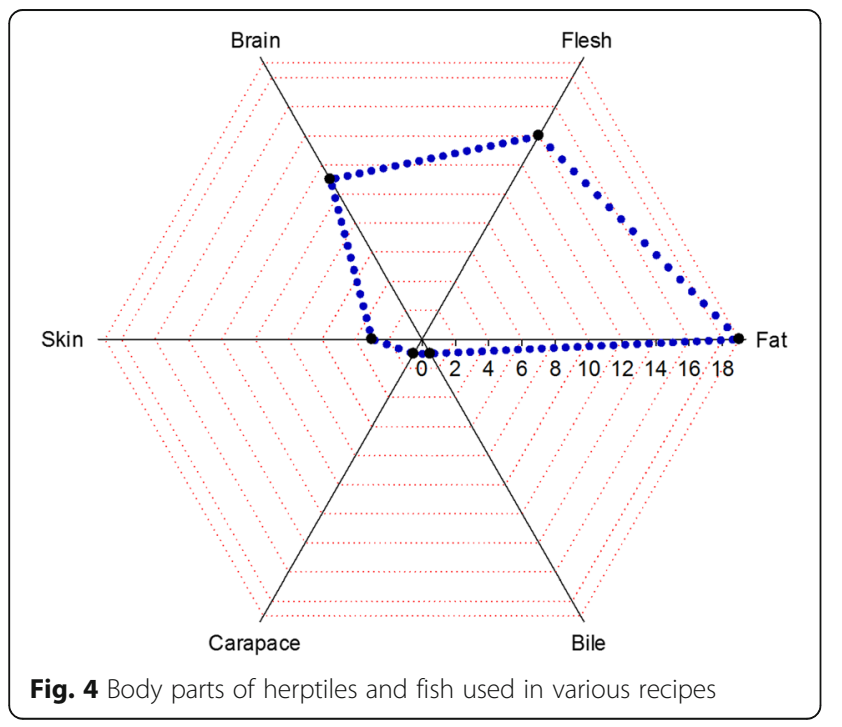

whereas $[48,59]$ it was reported that the same species is useful against ear pain, backache, joint pain, and headache. Local people used body fat, skin, and oil of the Indian cobra (Naja naja naja) to treat sciatica, snakebite, to improve eye sight, and as sex stimulant. This species has been reported to cure arthritis, cancer, leprosy, muscular pain, as aphrodisiac, and as antidote [48, 51, 52, 59]. Fat and oil of Russell's chain viper (Daboia russelii russelii) were used as a remedy for urine problem and hemorrhoids. However, in previous studies [50, 59, 87], different parts of this species have been reported as used against weak eye sight, urination, stool, flatus, and as anti-venom. Likewise, body fat and oil of the Sind valley saw snake viper (Echis carinatus sochureki) were used to treat joint pain, snakebite, weak eye sight, and to enhance sexual desire $[48,59]$.

Inhabitants of the study area preferred Ctenopharyngodon idella for the treatment of eyesight, night 


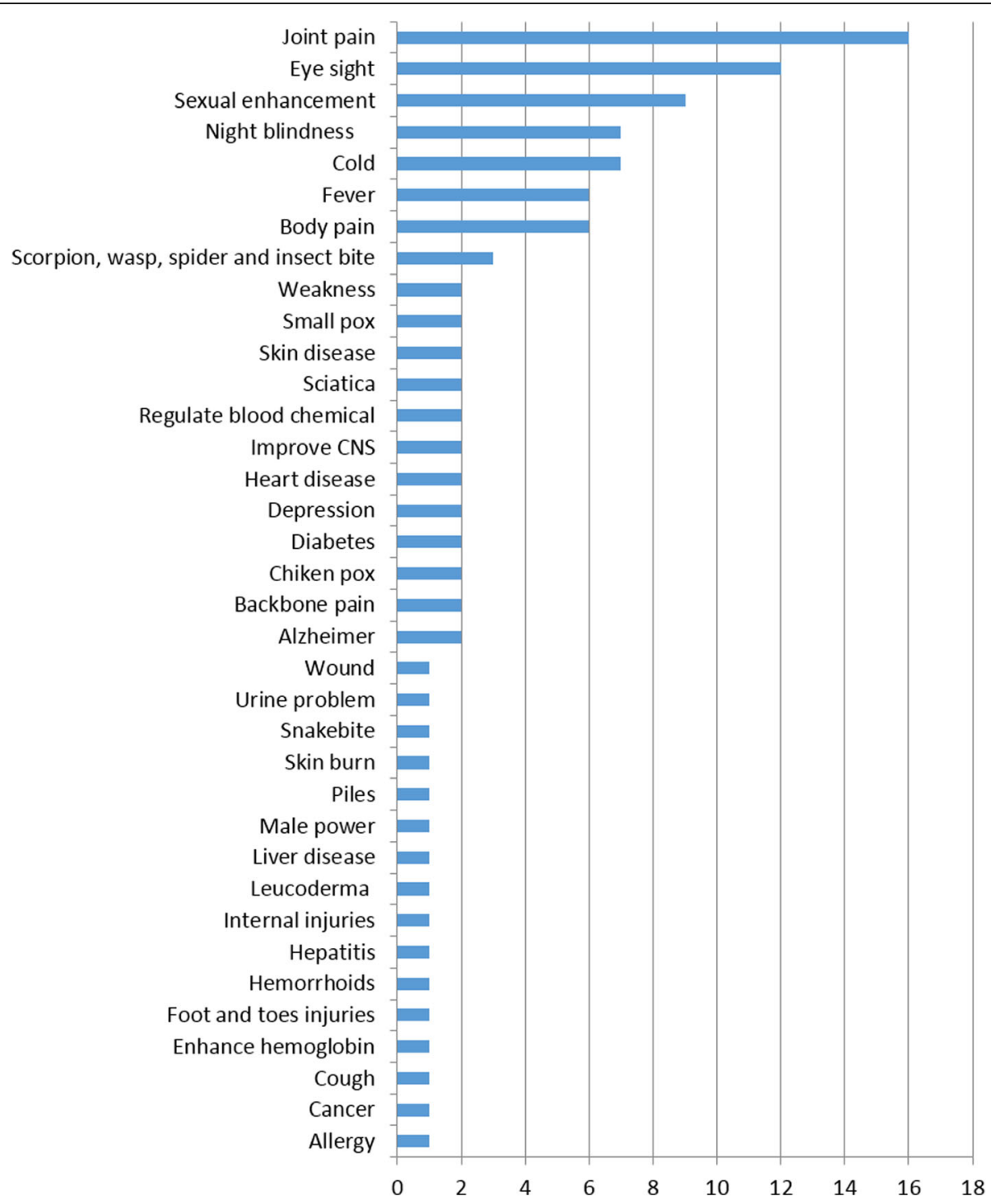

Fig. 5 Number of recipes and diseases treated

blindness, fever, cold, and joint pain, while the same species was reported to treat erectile disinfection, cold, to enhance memory, and sexual power and showed 0.20 similarity index with previous reports [31, 48]. Our findings revealed that Cyprinus carpio and Cirrhinus mrigala were effective against weak eyesight, night blindness, fever, cold, and joint pain. In previous studies [48, 60], C. carpio has been reported as used for CNS, erysipelas, lumbago, to enhance memory, enhance energy, sexual power, to reduce overweight, and against cold and has depicted similarity index $=0.20$. Likewise, $C$. mrigala was reported to reduce weight, to treat joint pain, to enhance memory and sexual power, to provide energy, and to treat against cold [48, 59]. Labeo rohita and L. calbasu were used for the treatment of joint pain, body pain, sexual potency, eyesight, depression, diabetes, Alzheimer, and heart diseases.
Similarly, the fish species Gibelion catla, Rita rita, Puntius sophore, and Heteropneustes fossilis were used to enhance hemoglobin, regulate blood chemistry, joint pain, sexual potency, improve CNS, cold, and have highest similarity index 1 with previous reports [47, 48, 59, 61].

The ethnomedicinal uses of eight herptiles, i.e., $A$. gangeticus, A. hurum, E. macularius, $V$. bengalensis, $P$. molurus, E. johnii, P. mucosus mucosus, D. russelii russelii and five fish species including H. molitrix, C. reba, L. dero, $M$. armatu, and P. ticto were reported for the first time from this region, and showed zero similarity with other studies. Among herptiles, H. tigerinus, D. stomaticus, and P. mucosus mucosu and in fishes L. rohita, W. $a t t u$, and C. reba were top ranked with maximum informant reports, frequency of citations, and rank order priority. 


\section{Cultural values of herptiles and fish}

Cultural values of the reported species of herptiles and fish are given in Table 2. Local people of the study area used the skin of the black cobra in magic. Likewise, different species of snakes like the Indian cobra (Naja naja naja), Indian rat snake (Ptyas mucosus mucosus), and buff striped keelback snake (Amphiesma stolatum) were used for pleasure of the public such as the mongoose competition with a snake. According to local informants, the presence of the yellow belly common house gecko (Hemidactylus flaviviridis) in a home is considered as bad omen for residents. The Bengal monitor (Varanus bengalensis) is tight knot with rope and with help of that rope a person can climb walls. Fish species were not only used in the treatment of various diseases but also as nutritious food. Local inhabitants used fish flesh as bait for varieties of fish species from rivers, as reported earlier by [88].

Inhabitants of the study area used different species of fish for commercial purposes. Likewise, Indian soft shell, Peacock soft shell, Indian Flap-shelled Turtle, and Indian Bullfrog were captured and sold for lab practice. Only four species of herptiles such as Indian soft shell (Aspideretes gangeticus), Peacock soft shell (Aspideretes hurum), Indian Flap-shelled Turtle (Lissemys punctata andersoni), and common leopard gecko (Eublepharis macularius) were exported from the area, and are used as food and for medicines. Two species of fish, i.e., Bronze featherback (Notopterus Notopterus) and Indian glassy fish (Parambassis ranga), are ornamental fish for aquaria.

The animal species reported by the maximum number of respondents were frequently used to treat various diseases, and exhibited high FC (Frequency of Citation) ranging from 5 to 90 (Fig. 6), i.e., Labeo rohita (rohu) had a maximum FC (90), followed by Wallago attu (wallago catfish) and Cirrhinus reba (Reba carp) (68 and 67, respectively).

The fidelity level is utilized to recognize species that are commonly favored by people to treat different diseases [89, 90]. The FL of herptile and fish species in this study varied from 2.9 to $100 \%$ (Table 2). Nine species, including $U$. hardwickii (Indus spiny-tail lizard), C. idella (grass carp), H. molitrix (silver carp), L. dero (dero), C. mrigala (mrigal carp), C. reba (reba carp), L. rohita (rohu), L. calbasu (orange fin labeo), and P. ticto (ticto barb) which were used for sexual potency, fever, cold, and to treat eyesight, body pain, and joint disorders, depicted 100\% FL (Fig. 5). These findings indicate the prevalence of particular diseases in the area that were cured with species having high FL. The animal species with maximum FL were highly used in the area, as compared to species having low FL. The FL of herptile and fish species was documented for the first time, and species with highest FL might be subjected to in-depth compositional analysis and bioactivities in pharmaceutical industries, as possible sources to manufacture novel drugs.

The relative popularity level (RPL) of the reported species is given in Table 2. Both herptile and fish species were classified as popular and unpopular categories based on RPL (Fig. 7), which were comparable to $[43,44]$. During the study, we noted that C. idella, C. carpio, H. molitrix, C. reba, C. mrigala, L. rohita, L. calbasu, L. dero, G. catla, C. punctata, C. marulius, $O$. niloticus, $R$. rita, B. bagarius, $M$. armatus, and $W$.

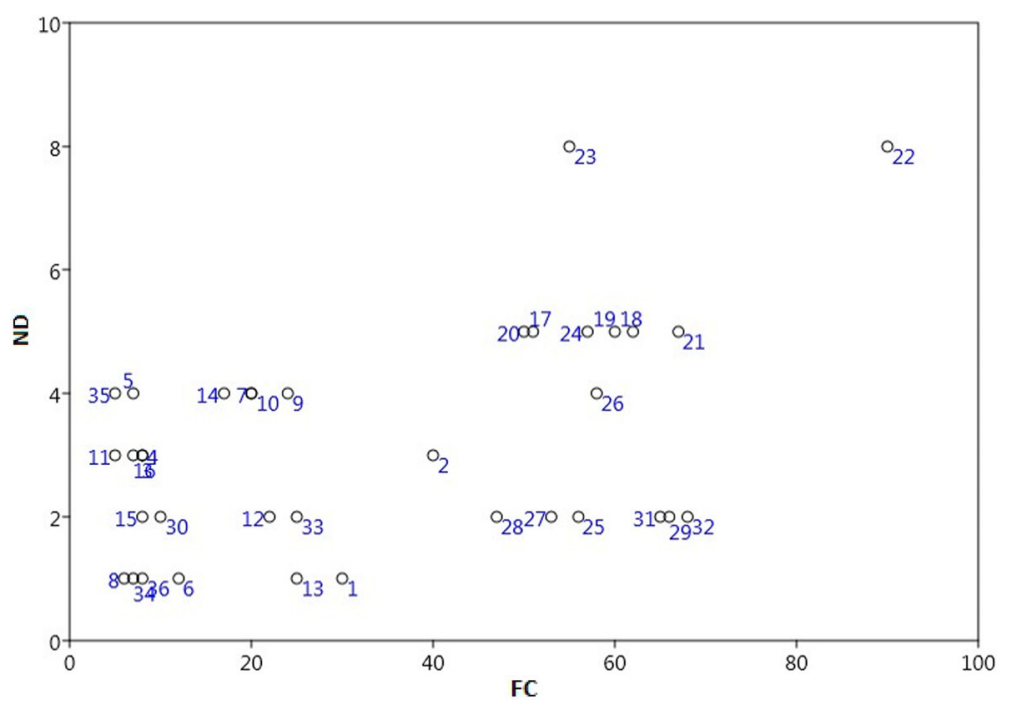

Fig. 6 The relationship between numbers of diseases (ND) and frequency of citation (FC) in the study area 


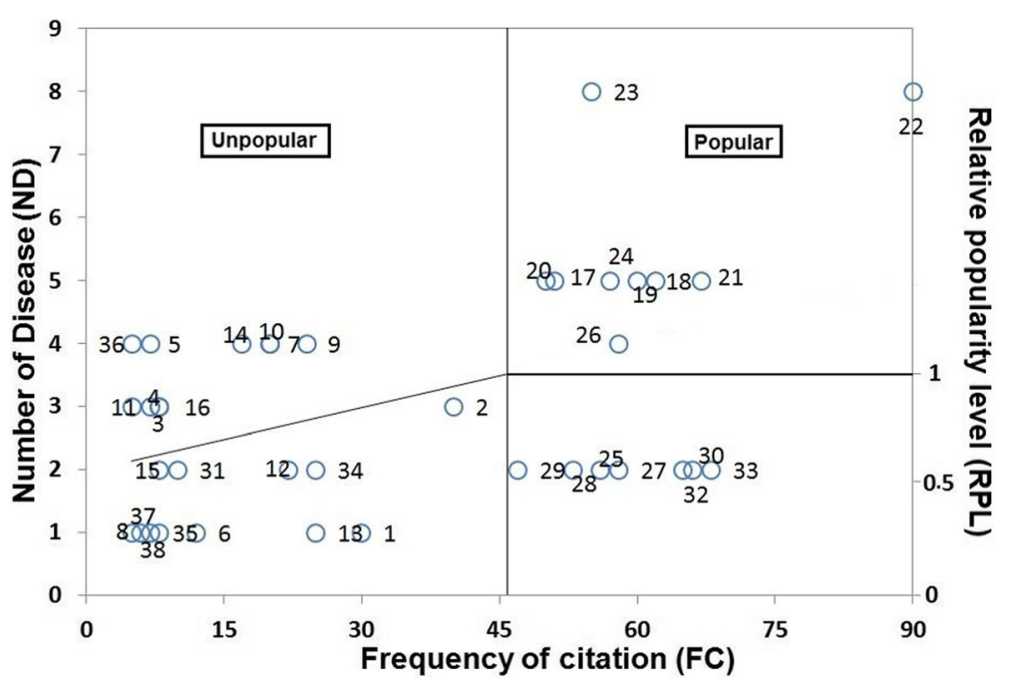

Fig. 7 The relationship between informant numbers and the percentage of informants who argued similar use for that herpetofauna and fish; circled numbers show herpetofauna and fish names as given in Table 1

attu were most popular with $\mathrm{RPL}=1.0$ while all other species were ranked as unpopular. The high popularity of these species might be attributed to their high efficiency utilized as medicine.

The healing potential of herptile and fish species was observed using FL values, while rank order priority (ROP) was utilized to assign a suitable grade to all species with various FL values. The measured levels of ROP of each herptile and fish species are cited in Table 3. The ROP of seven species used to treat different diseases was above 100 . These species were C. idella (eyesight and cold); C. carpio (fever); $H$. molitrix and C. reba (fever and cold); and L. rohita, L. calbasu, and $L$. dero used to treat joint pain, fever, and cold and for sexual potency. A decrease in ROP may be due to the decline in the popularity of ethnomedicinal and ethnocultural uses of fauna among local peoples. Moreover, the respondents of the rural area have more information and interaction with cultural and medicinal uses of animals compared to urban areas. The findings of the present survey were analogue to previous results for medicinal species of animal species in Palestine [44].

This study, for the first time, reported the use of flesh ash, fat, and oil of Aspideretes gangeticus (for skin diseases and sexual potency), Aspideretes hurum (backbone/joint pain), flesh ash of Calotes versicolor (for foot and toe injuries), fat oil of Daboia russelii russelii (to treat urinary problems and hemorrhoids), brain oil of Hypophthalmichthys molitrix (to improve eyesight, night blindness, and to treat fever, cold, joint pain). In addition, the brain oil of Cirrhinus reba and Labeo dero was used to treat eyesight, night blindness, fever, cold, and joint pain; the flesh of Mastacembelus armatus was used to improve sexual potency and body weakness; the brain of Pethia ticto was used to treat night blindness, eyesight, and to develop central nervous system; and the brain of Gagata cenia was used to treat urinary problems (Table 1).

Zoonoses with a wildlife reservoir are a major public health issue, affecting the whole world. Various pathogens and different modes of transmission are present, and many variables impact the epidemiology of different zoonoses. The recognition and importance of wildlife as a reservoir of zoonoses are increasing [91, 92]. The prevalence of transmission of disease-producing driving forces from fish to humans is however very low. In general, humans contract fish-borne disease through ingestion of tissues, or by contamination of the skin [93]. Human sensitivity to amphibian proteins in a laboratory setting is rare. It remains possible, however, to become sensitized to amphibian proteins through inhalation or skin contact [94].

\section{Conservation status of the reported species}

Knowing the background of interaction and exploitation between humans and natural resources is vital for the implementation and development of animal and landscape conservation strategies [95]. Ethnozoological studies provide necessary information and contribute significantly to animal conservation because in addition to incorporating biological factors, and providing traditional knowledge on medicinal and cultural values of animal species in any area, such studies also cover 
cultural, social, economic and traditional roles of fauna in human civilization [96].

Based on the cultural uses of herptiles and fish species (Table 2), it was observed that $47 \%$ of the reported species are listed as least concern (LC), 44\% are not evaluated (NE), 0.04\% species (i.e., Indian soft shell, Peacock soft shell, Rock pathon, and Common carp) are vulnerable (V), and $0.03 \%$ species (i.e., Silver carp, Foji Khaga, and wallago catfish) are listed as near threatened (NT) globally by the International Union of Conservation of Nature (IUCN). Interestingly, most of the herptiles and fish species (74/91\%) showed threats, and only $9 \%$ of the species were listed as threatened by IUCN as mentioned above. Use of animal species in traditional therapies and for cultural purpose by humans is not the only threat to animal biodiversity in any region. Factors also include changes in climate and various types of interactions in an ecosystem, i.e., food chain, food webs also contribute significantly in threatening animal population and diversity [96, 97]. Given the great need to find solutions to deal with the current crisis of biodiversity loss [98], more specifically that of animal species, it is obligatory to adopt strategies that address the problem in all its complexity. And for this, ethnozoology presents itself as an interdisciplinary tool, approaching the issue in an additional comprehensive method [99].

\section{Principal component analysis}

The ethnomedicinal data were analyzed through principal component analysis (PCA), which allowed for the ordination of plots in terms of three variables viz.
Informant of major ailment (IMA), fidelity level (FL), and rank order priority (ROP). The result of the PCA showed the sum of all the eigenvalues with total inertia of 3105.67. The first eigenvalue was high (2881.04) which showed high gradient strength in distribution of indigenous knowledge along the first axis (PC1). The total variation explained along this axis is (92.77\%). The first two axes of the principal component analysis showed 99.99\% variation in samples (component 1: 92.77\%; component 2: 7.23\%); therefore, only two axes were considered in Fig. 8. The variables IMA $(r=$ $0.33506)$, FL $(r=0.57662)$, and ROP $(r=0.74514)$ positively correlated with first axis (PC 1) while IMA $(r=$ $0.23734)$ and ROP $(r=-0.52551)$ was negatively correlated with component 2 and FL $(r=0.81701)$ was positively correlated with component 2 (PC 2), which were comparable with previous studies [100].

\section{Conclusion}

Traditional uses of various herptiles and fish species were recorded, and to the best of our knowledge, the ethno-pharmacological applications of 11 herptiles and seven fish species were reported for the first time from this region. Our findings revealed that the indigenous communities of the study possess significant traditional knowledge because of their strong relation with the nearby fauna. These results could be valuable for sustainable utilization and conservation of animal species. Additionally, detailed investigations on pharmacologically active substances and in vitro and/or in vivo of biological activities of compounds from herptiles and fish species with highest FL and FC could be interesting for

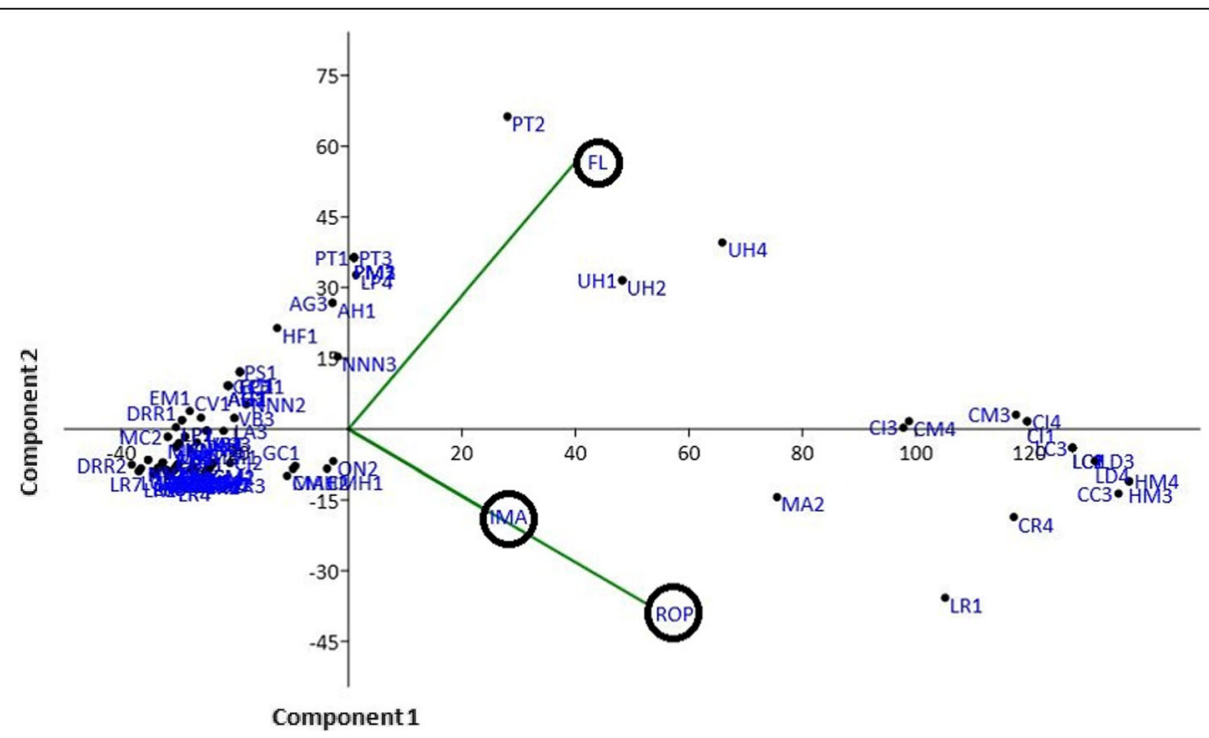

Fig. 8 Principal components analysis (PCA) (code are present in Table 1). The positions of the arrows relative to components 1 and 2 show how strongly independent variables (IMA, ROP and RI) are correlated 


\section{the development of novel animal-based drugs to treat various health disorders.}

\section{Acknowledgement}

Authors are highly thankful for the local people of river Chenab area for sharing valuable information

\section{Authors' contributions}

MA and AMA designed the project, were involve in data collection and analysis, and prepared final draft of the manuscript, MU, MSA, and KI helped in the statistical analysis and manuscript revision while AMK assisted in species identification. The author(s) read and approved the final manuscript.

\section{Funding}

This study was conducted purely on self-finance basis without and financia support.

\section{Availability of data and materials}

All data have already been included in the manuscript.

\section{Ethics approval and consent to participate}

Present study is purely based on filed survey instead of human or animal trails. Therefore, ethical approval and consent to participate is not applicable. However, formal consent was taken from informants regarding data collection and publication. In addition, ethical guidelines of the International Society of Ethnobiology (http://www.ethnobiology.net/) were strictly followed.

\section{Consent for publication}

Present paper does not contain any individual's person data; therefore, this section is not applicable to our study.

\section{Competing interests}

The authors declare that they have no conflict of interest

\section{Author details}

'Department of Zoology, Women University of Azad Jammu and Kashmir, Bagh, Pakistan. ${ }^{2}$ Department of Environment Sciences, COMSATS University Islamabad, Abbottabad Campus, Abbottabad 22060, Pakistan. ${ }^{3}$ School of Agriculture and Biology, Shanghai Jiao Tong University, Shanghai, China. ${ }^{4}$ Department of Botany, Women University of Azad Jammu and Kashmir, Bagh, Pakistan. ${ }^{5}$ Department of Zoology, University of the Punjab, Lahore, Pakistan

\section{Received: 7 February 2020 Accepted: 8 May 2020}

Published online: 24 June 2020

\section{References}

1. Kaplan H, Hill K, Lancaster J, Hurtado AM. A theory of human life history evolution: diet, intelligence, and longevity. Evol Anthropol Issues News Rev. 2000;9:156-85.

2. Alves RR, Rosa IL, Neto NAL, Voeks R. Animals for the gods: magical and religious faunal use and trade in Brazil. Hum Ecol. 2012;40:751-80

3. Altaf M, Javid A, Umair M, lqbal KJ, Rasheed Z, Abbasi AM. Ethnomedicinal and cultural practices of mammals and birds in the vicinity of river Chenab, Punjab-Pakistan. J Ethnobiol Ethnomed. 2017;13:1-24

4. Ali A. Survey of marine fishes at Ibrahim Hyderi (Jetty), KarachiPakistan. $J$ Wildl Ecol. 2017;1:42-7.

5. Ali A. Survey of marine fishes at Keti Bunder, district Thatha, Pakistan. J Wild Ecol. 2017;1:36-42.

6. Haider R, Altaf M, Rasheed Z, Rauf K, Mumtaz B, Altaf M, Shabir M, Hakeem F, Iftikhar A. Assessment of behavioral study, human activities impacts and interaction with white cheeked bulbul (Pycnonotus leucotis) in district Bagh, Azad Jammu and Kashmir, Pakistan. J Wildl Ecol. 2017:1:17-24.

7. Hakeem F, Altaf M, Manzoor S, Rauf K, Mumtaz B, Bashir M, Haider R, Farooq SI, Safdar L, Altaf M. Assessment of behavioral study, human activities impacts and interaction with Streak laughingthrush (Trochalopteron lineatum) in district Bagh, Azad Jammu and Kashmir-Pakistan. J Wildl Ecol. 2017;1:1-7.

8. Alves RR, Alves HN. The faunal drugstore: Animal-based remedies used in traditional medicines in Latin America. J Ethnobiol Ethnomed. 2011;7:9.
9. Alves RRN, Souto WMS. Ethnozoology: a brief introduction. Ethnobiol Conserv. 2015:4

10. Beetz A, Uvnäs-Moberg K, Julius H, Kotrschal K. Psychosocial and psychophysiological effects of human-animal interactions: the possible role of oxytocin. Front Psychol. 2012;3:234

11. Santos-Fita D, Costa-Neto E, Cano-Contreras E, Costa Neto E, Santos Fitas D, Vargas CM. El quehacer de la etnozoología. Man Etnozool. 2009:23-44.

12. Hajdari A, Pieroni A, Jhaveri M, Mustafa B, Quave C. Ethnomedical remedies among Slavic speaking people in South Kosovo. J Ethnobiol Ethnomed. 2018;7.

13. Martínez GJ. Use of fauna in the traditional medicine of native Toba (qom) from the Argentine Gran Chaco region: an ethnozoological and conservationist approach. Ethnobiol Conserv. 2013;2.

14. Altaf M, Javid A, Khan AM, Khan MSH, Umair M, Ali Z. Anthropogenic impact on the distribution of the birds in the tropical thorn forest, Punjab, Pakistan. J Asia Pac Biodivers. 2018.

15. Altaf M. Assessment of Avian and Mammalian Diversity at Selected Sites along river Chenab in Wildlife and Ecology. Lahore-Pakistan: University of Veterinary and Animal Sciences; 2016. p. 197.

16. Rômulo RNA, Gentil APF, Kleber SV, Wedson MSS, Lívia ETM, Paulo FGPM, Waltécio dOA, Washington LSV. A zoological catalogue of hunted reptiles in the semiarid region of Brazil. J Ethnobiol Ethnomed. 2012;8:1-29.

17. Alves RRN, das Graças Gerônimo Oliveira M, Barboza RRD, Lopez LCS. An ethnozoological survey of medicinal animals commercialized in the markets of Campina Grande, NE Brazil. Hum Ecol Rev. 2010:11-7.

18. Ferreira FS, Albuquerque UP, Coutinho HDM, Almeida WO, Alves RRN. The trade in medicinal animals in northeastern Brazil. Evid Based Complement Altern Med. 2012:2012.

19. Ferreira FS, Brito SV, de Oliveira AW, Alves RRN. Conservation of animals traded for medicinal purposes in Brazil: Can products derived from plants or domestic animals replace products of wild animals? Reg Environ Chang. 2016:16:543-51.

20. Hussain A, ASHRAF M, Altaf M, Khan WA, Akmal M, Qazi J. Relative diversity and threats to commercially important fishes of the Ravi, Pakistan. Biologia. 2015:145-9.

21. Khan MS. Herpetology of habitat types of Pakistan. Pak J Zool. 1999:31:275-89.

22. Alves R, Rosa IL. Zootherapeutic practices among fishing communities in North and Northeast Brazil: A comparison. J Ethnopharmacol. 2007:111:82-103.

23. Ferreira FS, Brito SV, Ribeiro SC, Saraiva AA, Almeida WO, Alves RR. Animalbased folk remedies sold in public markets in Crato and Juazeiro do Norte, Ceará, Brazil. BMC Complement Altern Med. 2009:9:17.

24. Kottelat $\mathrm{M}$, Whitten $\mathrm{T}$. Freshwater biodiversity in Asia: with special reference to fish, vol. 343: Place World Bank Publications: 1996.

25. Khan MS. Amphibian and Reptiles of Pakistan: Krieger Publisher Company; 2006.

26. Khan MS. Checklist of amphibians of Pakistan. Pak J Wildl. 2010;1.

27. Dudgeon $D$. An inventory of riverine biodiversity in monsoonal Asia: present status and conservation challenges. Water Sci Technol. 2002:45:11-9.

28. Froese R, Pauly D FishBase. 2017. http://www.fishbase.org version. Accessed 10 Apr 2017.

29. Muhammad N, Khan AM, Iqbal KJ, Haider MS, Ashraf S, Ansari ZS, Chattha SA, Abbasi AR, Yaqoob M. Assessment of distribution and ethnocultural uses of the Baringo tilapia (Oreochromis niloticus) in Punjab, Pakistan. J Wildl Ecol. 2017;1:7-13.

30. Muhammad N, Khan AM, Umair M, Qazi A, Yaqoob M, Ashraf S, Khan Q Farooq M. Assessment of distribution and ethnocultural uses of the Sol (Channa marulius) in Punjab, Pakistan. J Wildl Ecol. 2017;1:35-41.

31. Muhammad N, Umair M, Khan AM, Abbasi AR, Khan Q, Khan A, Awan MZ. Assessment of the diversity and ethno-medicinal uses of the carps in Punjab, Pakistan. J Wildl Ecol. 2017;1:52-60.

32. Rauf K, Altaf M, Mumtaz B, Altaf M, Haider R, Safeer B, Farooq SI, Safdar L, Manzoor M, Yasrub S, Bashir SM, Iftikhar A. Assessment of behavior, distribution, ecology and interaction study of Cinnamon Tree Sparrow (Passer rutilans) in district Bagh-Pakistan. J Wild Ecol. 2017:1:43-9.

33. Siddiqi TA, Tahir-Kheli S. Water and Security in South Asia; 2004. p. 234.

34. Sheikh MS. District Pre-Investment Study - 2012, Gujranwala., G.o. Punjab, Editor; 2012. p. 1-376

35. Sheikh MS. District Pre-Investment Study - 2012, Gujrat., G.o. Punjab, Editor; 2012. p. 1-28.

36. Sheikh MS. District Pre-Investment Study - 2012, Sialkot. G.o. Punjab, Editor; 2012. p. 1-31. 
37. Altaf M, Javid A, Khan AM, Hussain A, Umair M, Ali Z. The status of fish diversity of river Chenab, Pakistan. J Anim Plant Sci. 2015;25:564-9.

38. Altaf M, Javid A, Irfan, Munir M, Ashraf S, Umair M, Iqbal K, Khan A, Ali Z. Diversity of wild mammalian fauna of Chenab riverine forest, Punjab, Pakistan. J Anim Plant Sci. 2014;24:1342-7.

39. Khan MS. Amphibians and reptiles of Pakistan: Krieger Publishing Company; 2006.

40. Mirza MR. Fresh water Fishes of Pakistan: Urdu Science Board; 2004.

41. Tardío J, Pardo-de-Santayana M. Cultural importance indices: a comparative analysis based on the useful wild plants of Southern Cantabria (Northern Spain) 1. Econ Bot. 2008;62:24-39.

42. Alexiades MN, Sheldon JW. Selected guidelines for ethnobotanical research: a field manual: New York Botanical Garden; 1996.

43. Friedman J, Yaniv Z, Dafni A, Palewitch D. A preliminary classification of the healing potential of medicinal plants, based on a rational analysis of an ethnopharmacological field survey among Bedouins in the Negev Desert, Israel. J Ethnopharmacol. 1986;16:275-87.

44. Ali-Shtayeh MS, Yaniv Z, Mahajna J. Ethnobotanical survey in the Palestinian area: a classification of the healing potential of medicinal plants. Ethnopharmacol. 2000;73:221-32.

45. Hammer O, Harper DAT, Ryan PD. Past: Palaeontological statistical software package for education and data analysis. Palaeontol Electron. 2001;4:9.

46. Khan FM, Chaudhry H, Mustafa YS, Ahmad W, Farhan HM. Ethno-Veterinary Zoo-Therapies and occult practices in greater Cholistan desert (Pakistan). Sci Int. 2011;23:241-3.

47. Borah MP, Prasad SB. Ethnozoological study of animals based medicine used by traditional healers and indigenous inhabitants in the adjoining areas of Gibbon Wildlife Sanctuary, Assam, India. J Ethnobiol Ethnomed. 2017;13:1-13.

48. Altaf M, Umair M, Abbasi AR, Muhammad N, Abbasi AM. Ethnomedicinal applications of animal species by the local communities of Punjab, Pakistan. J Ethnobiol Ethnomed. 2018;14:1-25.

49. Lohani U. Traditional uses of animals among jirels of Central Nepal. Ethno Med. 2011;5:115-24.

50. Benarjee G, Srikanth K, Ramu G, Ramulua K. Ethnozoological study in a tropical wildlife sanctuary of Eturunagaram in the Warangal district, Andhra Pradesh. Ind J Trad Knowledge. 2010;9:701-4.

51. Bagde N, Jain S. An ethnozoological studies and medicinal values of vertebrate origin in the adjoining areas of Pench National Park of Chhindwara District of Madhya Pradesh, India. Int J Life Sci. 2013;1:278-83.

52. Bagde N, Jain S. Study of traditional man-animal relationship in chhindwara district of Madhya Pradesh, India. J Glob Biosci. 2015;4:1456-63.

53. Vijayakumar S, Prabhu S, Yabesh JM, Prakashraj R. A quantitative ethnozoological study of traditionally used animals in Pachamalai hills of Tamil Nadu, India. J Ethnopharmacol. 2015.

54. Mishra N, Rout S, Panda T. Ethno-zoological studies and medicinal values of Similipal Biosphere Reserve, Orissa, India. Afr J Pharm Pharmacol. 2011;5:6-11.

55. Vijayakumar S, Yabesh JM, Prabhu S, Ayyanar M, Damodaran R. Ethnozoological study of animals used by traditional healers in Silent Valley of Kerala, India. J Ethnopharmacol. 2015;162:296-305.

56. Padmanabhan P, Sujana K. Animal products in traditional medicine from Attappady hills of Western Ghats. Indian J Tradit Knowl. 2008;7:326-9.

57. Chakravorty J, Meyer-Rochow VB, Ghosh S. Vertebrates used for medicinal purposes by members of the Nyishi and Galo tribes in Arunachal Pradesh (North-East India). J Ethnobiol Ethnomed. 2011;7:1.

58. Lalmuanpuii J, Rosangkima G, Lamin H. Ethno-medicinal practices among the Mizo ethnic group in Lunglei district, Mizoram. Sci Vis. 2013;13:24-34.

59. Arshad M, Ahmad M, Ahmed E, Saboor A, Abbas A, Sadiq S. An ethnobiological study in Kala Chitta hills of Pothwar region, Pakistan: multinomial logit specification. J Ethnobiol Ethnomed. 2014;10:13.

60. Vallejo JR, González JA. Fish-based remedies in Spanish ethnomedicine: a review from a historical perspective. J Ethnobiol Ethnomed. 2014;10:37.

61. Saikia K, Ahmed R. Wetland fish biodiversity of Majuli river island (India) and their medicinal values. Clarion. 2012;1.

62. Deb AK, Emdad HC. '< i $>$ Every mother is a mini-doctor $</ \mathrm{i}>$ ': Ethnomedicinal uses of fish, shellfish and some other aquatic animals in Bangladesh. J Ethnopharmacol. 2011;134:259-67.

63. Teronpi V, Singh H, Tamuli A, Teron R. Ethnozoology of the Karbis of Assam, India: Use of ichthyofauna in traditional health-care practices. Anc Sci Life. 2012;32:99.
64. Mahawar MM, Jaroli D. Traditional zootherapeutic studies in India: a review. J Ethnobiol Ethnomed. 2008;4:17.

65. Gupta T, Dey M. Ichthyotheropy: use of fishes as medicine by ethnic Karbi people of Assam, India; 2017.

66. Benítez G. Animals used for medicinal and magico-religious purposes in western Granada Province, Andalusia (Spain). J Ethnopharmacol. 2011;137: $1113-23$.

67. Barros FB, Varela SA, Pereira HM, Vicente L. Medicinal use of fauna by a traditional community in the Brazilian Amazonia. J Ethnobiol Ethnomed. 2012;8:37.

68. Mawla F, Khatoon S, Rehana F, Jahan S, Shelley M, Hossain S, Haq WM, Rahman S, Debnath K, Rahmatullah M. Ethnomedicinal plants of folk medicinal practitioners in four villages of Natore and Rajshahi districts, Bangladesh. Am Eur J Sustain Agric. 2012;6:406-16.

69. Mukti M, Ahmed A, Chowdhury S, Khatun Z, Bhuiyan P, Debnath K, Rahmatullah M. Medicinal plant formulations of Kavirajes in several areas of Faridpur and Rajbari districts, Bangladesh. Am-Eur J Sustain Agr. 2012;6:234-47.

70. Deb AK, Haque CE. Every mother is a mini-doctor: Ethnomedicinal uses of fish, shellfish and some other aquatic animals in Bangladesh. J Ethnopharmacol. 2011;134:259-67.

71. Jaroli D, Mahawar MM, Vyas N. An ethnozoological study in the adjoining areas of Mount Abu wildlife sanctuary, India. J Ethnobiol Ethnomed. 2010;6:6.

72. Alves RR, Neto NAL, Brooks SE, Albuquerque UP. Commercialization of animal-derived remedies as complementary medicine in the semi-arid region of Northeastern Brazil. J Ethnopharmacol. 2009;124:600-8.

73. Wilson L. Fats and oils for optimum health. The Center for Development; 2015.

74. Kabir M, Skurnik G, Naour N, Pechtner V, Meugnier E, Rome S, QuignardBoulangé A, Vidal H, Slama G, Clément K. Treatment for 2 mo with $n-3$ polyunsaturated fatty acids reduces adiposity and some atherogenic factors but does not improve insulin sensitivity in women with type 2 diabetes: a randomized controlled study. Am J Clin Nutr. 2007;86:1670-9.

75. Esposito K, Marfella R, Ciotola M, Di Palo C, Giugliano F, Giugliano G, D'Armiento M, D'Andrea F, Giugliano D. Effect of a Mediterranean-style diet on endothelial dysfunction and markers of vascular inflammation in the metabolic syndrome: a randomized trial. JAMA. 2004;292:1440-6.

76. Morgan DR, Dixon LJ, Hanratty CG, El-Sherbeeny N, Hamilton PB, McGrath LT, Leahey WJ, Johnston GD, McVeigh GE. Effects of dietary omega-3 fatty acid supplementation on endothelium-dependent vasodilation in patients with chronic heart failure. Am J Cardiol. 2006;97:547-51.

77. Mori TA, Bao DQ, Burke V, Puddey IB, Watts GF, Beilin LJ. Dietary fish as a major component of a weight-loss diet: effect on serum lipids, glucose, and insulin metabolism in overweight hypertensive subjects. Am J Clin Nutr. 1999;70:817-25.

78. FAO. The Composition of Fish. 2001. FAO and Support unit for International Fisheries and Aquatic Research (SIFAR).

79. Kimura S. Wide distribution of the skin type I collagen alpha 3 chain in bony fish. Comp Biochem Physiol B. 1992;102:255-60.

80. Sivakumar P, Arichandran R, Suguna L, Mariappan M, Chandrakasan G. The composition and characteristics of skin and muscle collagens from a freshwater catfish grown in biologically treated tannery effuent water. J Fish Biol. 2000;56:999-1012.

81. Raman M, Gopakumar K. Fish Collagen and its Applications in Food and Pharmaceutical Industry: A Review. EC Nutr. 2018;13:752-67.

82. Erkan N, Bilen $\mathrm{G}$. Effect of essential oils treatment on the frozen storage stability of chub mackerel fillets. J Verbr Lebensm. 2010;5:101-10.

83. Alibardi L, Toni M. Cytochemical, biochemical and molecular aspects of the process of keratinization in the epidermis of reptilian scales. Prog Histochem Cytochem. 2006:40:73-134.

84. Balani K, Patel RR, Keshri AK, Lahiri D, Agarwal A. Multi-scale hierarchy of Chelydra serpentina: microstructure and mechanical properties of turtle shell. Je Mech Behav BiomedMater. 2011;4:1440-51.

85. Currey JD. Bones: structure and mechanics: Princeton University Press; 2013.

86. Calder PC, Yaqoob P. Omega-3 polyunsaturated fatty acids and human health outcomes. Biofactors. 2009:35:266-72.

87. Aloufi A, Eid E. Zootherapy: A study from the northwestern region of the kingdom of Saudi Arabia and hashemite kingdom of Jordan. Indian J Tradit Knowl. 2016;15:561-9.

88. del Valle YG, Naranjo EJ, Caballero J, Martorell C, Ruan-Soto F, Enríquez PL. Cultural significance of wild mammals in mayan and mestizo communities of the Lacandon Rainforest, Chiapas, Mixico. J Ethnobiol Ethnomed. 2015;11:1. 
89. Srithi K, Balslev H, Wangpakapattanawong P, Srisanga P, Trisonthi C. Medicinal plant knowledge and its erosion among the Mien (Yao) in northern Thailand. J Ethnopharmacol. 2009;123:335-42.

90. Bibi T, Ahmad M, Tareen RB, Tareen NM, Jabeen R, Rehman S-U, Sultana S, Zafar M, Yaseen G. Ethnobotany of medicinal plants in district Mastung of Balochistan province-Pakistan. J Ethnopharmacol. 2014;157:79-89.

91. Kruse H, Kirkemo A-M, Handeland K. Wildlife as source of zoonotic infections. Emerg Infect Dis. 2004;10:2067.

92. Van Vliet N, Moreno Calderón JL, Gomez J, Zhou W, Fa JE, Golden C, Nobrega Alves RR, Nasi R. Bushmeat and human health: assessing the evidence in tropical and sub-tropical forests. J Ethnobiol Ethnomed. 2017;6:1-45.

93. EHS. Zoonotic Diseases - Fish. In: ENVIronmental Health and Safety | Occupational Health: EHS Occupational Health Clinic; 2016

94. EHS. Zoonotic Diseases - Amphibians: EHS Occupational Health Clinic; 2016

95. Albuquerque UP, de Sousa DCP. Ethnobiology and Biodiversity Conservation, in Introduction to ethnobiology. Springer: Place. Published.

96. Alves RR. Relationships between fauna and people and the role of ethnozoology in animal conservation. Ethnobiol Conserv. 2012;1:1-69.

97. Alves RRN, Silva JS, da Silva Chaves L, Albuquerque UP. Ethnozoology and animal conservation, in Ethnozoology. Elsevier: Place. Published.

98. Boivin NL, Zeder MA, Fuller DQ, Crowther A, Larson G, Erlandson JM, Denham T, Petraglia MD. Ecological consequences of human niche construction: Examining long-term anthropogenic shaping of global species distributions. Proc Natl Acad Sci. 2016:113:6388-96.

99. Dickman AJ. Complexities of conflict: the importance of considering social factors for effectively resolving human-wildlife conflict. Anim Conserv. 2010 13:458-66.

100. Saunders CD. The emerging field of conservation psychology. Hum Ecol Rev. 2003:137-49.

\section{Publisher's Note}

Springer Nature remains neutral with regard to jurisdictional claims in published maps and institutional affiliations.

Ready to submit your research? Choose BMC and benefit from:

- fast, convenient online submission

- thorough peer review by experienced researchers in your field

- rapid publication on acceptance

- support for research data, including large and complex data types

- gold Open Access which fosters wider collaboration and increased citations

- maximum visibility for your research: over $100 \mathrm{M}$ website views per year

At BMC, research is always in progress.

Learn more biomedcentral.com/submissions 